\title{
Wreath Macdonald polynomials and the categorical McKay correspondence
}

\author{
Roman Bezrukavnikov and Michael Finkelberg \\ WITH AN APPENDIX BY VADIM VOLOGODSKY
}

To the memory of Andrei Zelevinsky

\begin{abstract}
Mark Haiman has reduced Macdonald Positivity Conjecture to a statement about geometry of the Hilbert scheme of points on the plane, and formulated a generalization of the conjectures where the symmetric group is replaced by the wreath product $\mathfrak{S}_{n} \ltimes(\mathbb{Z} / r \mathbb{Z})^{n}$. He has proven the original conjecture by establishing the geometric statement about the Hilbert scheme, as a byproduct he obtained a derived equivalence between coherent sheaves on the Hilbert scheme and coherent sheaves on the orbifold quotient of $\mathbb{A}^{2 n}$ by the symmetric group $\mathfrak{S}_{n}$.

A short proof of a similar derived equivalence for any symplectic quotient singularity has been obtained by the first author and Kaledin [2] via quantization in positive characteristic. In the present note we prove various properties of these derived equivalences and then deduce generalized Macdonald positivity for wreath products.
\end{abstract}

1 Introduction $\quad 164$

1.1

1.2 Acknowledgments

2 Localization of modules over the invariants in the Weyl algebra

2.1 Notations

2.2 Splitting bundles for Azumaya algebras

\subsection{Lagrangian components}

2.4 Localization for Verma modules for $\Gamma_{n}$-invariants in the Weyl algebra 
3 Spherical Cherednik algebras and localization in families 172

3.1 Quiver varieties and their quantization in mixed and positive characteristic

3.2 The family of Cherednik algebras and their localization

3.3 Verma modules

3.4 Supports of Verma modules

4 The proof of the main Theorem

4.1 Procesi bundle

4.2 Proof of Theorem 1.1

\subsection{Wreath Macdonald polynomials}

5 Errata to [2]

6 Appendix: Equivariant structure on rigid vector bundles 186

References

\section{Introduction}

The celebrated Macdonald Positivity Conjecture asserts that the entries of the matrix expressing the transformed Macdonald polynomials via Schur polynomials have non-negative coefficients.

The following approach to the conjecture originated from and was completed in the work of M. Haiman [15].

Let $\operatorname{Hilb}^{n}\left(\mathbb{A}^{2}\right)$ be the Hilbert scheme of $n$ points on the plane. The action of the two-dimensional torus $T=\mathbb{G}_{m}^{2}$ on $\mathbb{A}^{2}$ induces an action of $T$ on $\operatorname{Hilb}^{n}\left(\mathbb{A}^{2}\right)$. Let $\Lambda$ be the ring of symmetric polynomials. According to Haiman's program, one can identify the space $\Lambda\left[q^{ \pm 1}, t^{ \pm 1}\right]$ appearing in the Positivity Conjecture with the Grothendieck group $K^{0}\left(\operatorname{Coh}^{T}\left(\operatorname{Hilb}^{n}\left(\mathbb{A}^{2}\right)\right)\right)$ in such a way that the modified Macdonald polynomials correspond to classes of sky-scrapers at the fixed points of $T$, while Schur functions correspond to classes of indecomposable summands components of the Procesi bundle. The latter is a certain vector bundle on $\operatorname{Hilb}^{n}\left(\mathbb{A}^{2}\right)$ of rank $n$ ! carrying an action of the symmetric group and a compatible action of the ring of polynomials in $2 n$ variables. An explicit construction of this bundle is the crucial and 
laborious ingredient in Haiman's work. As a byproduct of this construction Haiman obtained an equivalence of derived categories:

$$
D^{b}\left(\operatorname{Coh}\left(\operatorname{Hilb}^{n}\left(\mathbb{A}^{2}\right)\right) \cong D^{b}\left(\operatorname{Coh}^{\mathfrak{S}_{n}}\left(\mathbb{A}^{2 n}\right)\right)\right.
$$

and an isomorphism between $\operatorname{Hilb}^{n}\left(\mathbb{A}^{2}\right)$ and the Hilbert quotient of $\mathbb{A}^{2 n}$ by the action of $\mathfrak{S}_{n}$.

The existence of a rank $n$ ! bundle with an action of $\mathfrak{S}_{n}$ and of the polynomial ring which induces a derived equivalence (1.1) has been proven in another, shorter way in [2] via quantization in positive characteristic.

The goal of the present note is to demonstrate that the information provided by [2] is sufficient to deduce the Positivity Conjecture, bypassing the explicit construction of the Procesi bundle. ${ }^{1}$ Furthermore, Haiman [16] has suggested an extension of the conjecture to the setting where $\mathfrak{S}_{n}$ is replaced by the wreath product $\mathfrak{S}_{n} \ltimes(\mathbb{Z} / r \mathbb{Z})^{n}$. We also confirm that previously unknown conjecture.

We should mention that another proof of the Positivity Conjecture independent of Haiman's construction of Procesi bundle has been obtained by I. Gordon [14] based on a result of V. Ginzburg [10]. This approach employs the theory of Hodge $D$-modules to pass from modules over a noncommutative algebra to modules over its commutative degeneration; in the present article this is achieved by virtue of the $p$-center phenomenon in the theory of $D$-modules in positive characteristic. There are other situations when the two apparently very different constructions lead to the same structures, see e.g. $[4, \S 0.2$; Remark 2.2.2.(2)]. It would be very interesting to find an explanation for this parallelism.

\section{1 .}

Let us formulate our result in more detail. We set $\Gamma=\mathbb{Z} / r \mathbb{Z}$. We consider the cyclic quiver $Q$ with the set of vertices $I=\left\{i_{0}, \ldots, i_{r-1}\right\} \cong \mathbb{Z} / r \mathbb{Z}$. Our basic field $\mathrm{k}$ will be either $\mathbb{C}$ or $\overline{\mathbb{F}}_{p}$ with $p \gg 0$. Given $I$-graded vector spaces $W, V$ of dimension vectors $\lambda_{0}=(1,0, \ldots, 0), \nu=\left(v_{0}, \ldots, v_{r-1}\right)$, we consider the Nakajima quiver varieties $\mathfrak{M}(W, V) \stackrel{\pi}{\longrightarrow} \mathfrak{M}_{0}(W, V)$ over k. It is well known

\footnotetext{
${ }^{1}$ Notice however that we do not propose an alternative proof of the isomorphism between $\operatorname{Hilb}^{n}\left(\mathbb{A}^{2}\right)$ and the Hilbert quotient of $\mathbb{A}^{2 n}$, which is one of the equivalent statements of the $n$ ! conjecture. Existence of such an isomorphism is equivalent to the fact that each fiber of the rank $n$ ! vector bundle is generated by an $\mathfrak{S}_{n}$ invariant vector under the action of the polynomial ring. We do not know how to deduce this property of the vector bundle by our methods.
} 
(see e.g. [16, Section 7.2.3] or $\left[13\right.$, Lemma 7.8]) that $\mathfrak{M}_{0}(W, V) \cong \mathbb{A}^{2 n} / \Gamma_{n}$ where $\Gamma_{n}$ is the wreath product $\mathbb{Z} / r \mathbb{Z} \imath \mathfrak{S}_{n}$, where $n$ depends on $\nu$ as follows. Let $\delta$ stand for the dimension vector $(1, \ldots, 1)$. Then we have a unique decomposition $\nu=\nu_{0}+n \delta$ where $\nu_{0}$ is the content vector of a certain $r$ core partition. Moreover, $\mathfrak{M}(W, V) \cong Y_{\Gamma, \nu}$, where $Y_{\Gamma, \nu}$ is a certain connected component of the fixed point set $\left(\operatorname{Hilb}^{m}\left(\mathbb{A}^{2}\right)\right)^{\Gamma}$ of a Hilbert scheme of points on $\mathbb{A}^{2}$. Here $m=v_{0}+\ldots+v_{r-1}[21, \S 4.2]$. Finally, $\pi$ is a symplectic semismall resolution of singularities.

According to the result of [2] we have an equivalence of derived categories $D^{b}\left(\operatorname{Coh}\left(Y_{\Gamma, \nu}\right)\right) \cong D^{b}\left(\operatorname{Coh}^{\Gamma_{n}}\left(\mathbb{A}^{2 n}\right)\right)$ provided that characteristic of $\mathrm{k}$ is zero or sufficiently large. Let $\mathcal{E} \in D^{b}\left(\operatorname{Coh}\left(Y_{\Gamma, \nu}\right)\right)$ be the image of $\mathcal{O} \otimes \mathrm{k}\left[\Gamma_{n}\right] \in$ $\operatorname{Coh}^{\Gamma_{n}}\left(\mathbb{A}^{2 n}\right)$ under the equivalence constructed in [2]. Then it is shown in loc. cit. that $\mathcal{E}$ is a vector bundle. It automatically carries an action of $\Gamma_{n}$, thus it can canonically be written as $\mathcal{E}=\oplus \rho \otimes \mathcal{E}_{\rho}$ where $\rho$ runs over the set of irreducible representations of $\Gamma_{n}$ and $\varepsilon_{\rho}$ is a vector bundle on $Y_{\Gamma, \nu}$.

Furthermore, $\mathcal{E}$ carries an action of the polynomial algebra $\mathrm{k}\left[x_{1}, \ldots, x_{n}, y_{1}, \ldots, y_{n}\right]=\mathcal{O}\left(\mathbb{A}^{2 n}\right)$. Let $\bar{\varepsilon}_{\rho}$ be the image of $\varepsilon_{\rho}$ in the sheaf of coinvariants $\mathcal{E} /\left(\sum_{i=1}^{n} x_{i} \mathcal{E}\right)$, and $\overline{\mathcal{E}}_{\rho}^{\prime}$ be the image of $\mathcal{E}_{\rho}$ in $\mathcal{E} /\left(\sum_{i=1}^{n} y_{i} \mathcal{E}\right)$.

Our main result is (in the notations and conventions introduced below in subsection 2.3.)

Theorem 1.1. If $\rho=\rho_{\boldsymbol{\lambda}}$ for a multi-partition $\boldsymbol{\lambda}$, then the set of $T$-fixed points in the support of $\overline{\mathcal{E}}_{\rho}$ consists of $\boldsymbol{\lambda}$ and some $\boldsymbol{\mu}$ satisfying $\boldsymbol{\mu} \preceq \boldsymbol{\lambda}$, while the set of $T$-fixed points in the support of $\bar{\varepsilon}_{\rho}^{\prime}$ consists of ${ }^{t} \boldsymbol{\lambda}$ and some $\boldsymbol{\mu}$ satisfying $\boldsymbol{\mu} \succeq{ }^{t} \boldsymbol{\lambda}$.

The proof of the Theorem appears in subsection 4.2. It is based on the localization result of [2], while the analysis of partial orders on the set of fixed points follows the results of I. Gordon [13]. Notice that Gordon worked in a different setting, where the characteristic of the base field is zero, but one of the deformation parameters for the deformation of $\mathcal{O}\left(\mathbb{A}^{2 n}\right)^{\Gamma_{n}}$ is also zero, so one still gets an algebra with a large center. To relate the two settings we include the equivalence of [2] in a family, see beginning of section 3 for further comments.

The next statement follows from Corollary 4.3.

Corollary 1.2. Conjecture [16, 7.2.19] holds.

Notice that notations of [16] differ from ours by swapping the role of letters $q$ and $t$; also condition (ii) of loc. cit. appears below in an equivalent form obtained by replacing $(-t)^{-i}$ by $(-t)^{i}$ and replacing $\Lambda^{i}(\mathfrak{h})$ by the dual space tensored by the sign representation, which is equivalent to replacing $\boldsymbol{\lambda}$ by $\boldsymbol{\lambda}^{\prime}$ (or ${ }^{t} \boldsymbol{\lambda}$ in our notation). 


\subsection{Acknowledgments}

We are grateful to V. Ginzburg, I. Gordon, W. van der Kallen and B. Webster for some very useful discussions. Special thanks go to I. Losev for expert critical comments which contributed to our understanding of the material and influenced the exposition.

This work was conceived when the authors enjoyed the hospitality and support of IAS at HUJI in the Fall 2010. R.B. was partially supported by NSF grant DMS-1102434. M.F. was partially supported by the RFBR grants 12-01-00944, 12-01-33101, 13-01-12401/13, and the AG Laboratory HSE, RF government grant, ag. 11.G34.31.0023.

\section{Localization of modules over the invariants in the Weyl algebra}

\subsection{Notations}

From now on we assume that in case $\mathrm{k}=\overline{\mathbb{F}}_{p}$, the characteristic $p$ is bigger than $\left|\Gamma_{n}\right|$. In that case, for an algebraic variety $X$ over $\mathrm{k}$ we will denote by $X^{(1)}$ its Frobenius twist, and by $\mathrm{Fr}: X \rightarrow X^{(1)}$ the Frobenius morphism. Note that in the presentation $\mathfrak{M}_{0}(W, V) \cong \mathbb{A}^{2 n} / \Gamma_{n}$ the vector space $U=\mathbb{A}^{2 n}$ carries a $\Gamma_{n}$-invariant symplectic form $\omega$. It gives rise to the Weyl algebra $W_{k}$ acted upon by $\Gamma_{n}$. According to [2, Lemma 6.1], for large $p$ the $\mathbf{W}_{k}^{\Gamma_{n}}-W_{k} \rtimes$ $\mathrm{k}\left[\Gamma_{n}\right]$-bimodule $\mathrm{W}_{\mathrm{k}}$ gives rise to the Morita equivalence $\mathrm{W}_{\mathrm{k}}^{\Gamma_{n}} \sim \mathrm{W}_{\mathrm{k}} \rtimes \mathrm{k}\left[\Gamma_{n}\right]$. We will denote $Y_{\Gamma, \nu}$ introduced in Section 1.1 by $Y$ for short, and if we want to stress the base field, we will denote it by $Y_{\mathrm{k}}$.

In case $\mathrm{k}=\overline{\mathbb{F}}_{p}$, the Frobenius twist $Y^{(1)}$ carries a sheaf $\mathrm{O}$ of Azumaya algebras such that $H^{i}\left(Y^{(1)}, \mathrm{O}\right)=0$ for $i>0$, and $H^{0}\left(Y^{(1)}, \mathrm{O}\right)=\mathrm{W}_{\mathrm{k}}^{\Gamma_{n}}([2$, Lemma 6.2], see also correction below in section 5). From the point of view of [7], $\mathrm{O}$ is the localization of the spherical subalgebra of the symplectic reflection algebra $H_{0}$ of $\left(\Gamma_{n}, U\right)$ (rational Cherednik algebra) for the zero value of parameters.

We choose a $\Gamma_{n}$-invariant Lagrangian vector subspace $L \subset U$. We will denote the categorical quotient $L / \Gamma_{n} \subset U / \Gamma_{n}$ by $\mathfrak{L}_{0} \subset \mathfrak{M}_{0}(W, V)$. We will denote the scheme-theoretic preimage $\pi^{-1}\left(\mathfrak{L}_{0}\right) \subset \mathfrak{M}(W, V)=Y$ by $\mathfrak{L} \subset Y$. The completions of our schemes along their subschemes will be denoted like $\widehat{Y}_{\mathfrak{L}}$. Note that the dilation action of $\mathbb{G}_{m}$ on $\operatorname{Hom}\left(V_{i}, V_{i \pm 1}\right)$ descends to the $\mathbb{G}_{m}$-action on $\mathfrak{M}_{0}(W, V)$, and then lifts to the $\mathbb{G}_{m}$-action on $Y$.

We will also need another commuting action of $\mathbb{G}_{m}$ on $Y$ : the hyperbolic one, preserving the symplectic form on $Y$. To distinguish it from 
the dilation action above, we will denote this copy of $\mathbb{G}_{m}$ by $\mathbb{G}_{m}^{h}$. So if a point $y$ of $Y$ is represented by a quadruple $\left(B_{i} \in \operatorname{Hom}\left(V_{i}, V_{i+1}\right), B_{i}^{\prime} \in\right.$ $\left.\operatorname{Hom}\left(V_{i}, V_{i-1}\right), x_{0} \in V_{0}, x_{0}^{*} \in V_{0}^{*}\right)$, and $c \in \mathbb{G}_{m}^{h}$, then $c y$ is represented by $\left(c B_{i}, c^{-1} B_{i}^{\prime}, x_{0}, x_{0}^{*}\right)$.

\subsection{Splitting bundles for Azumaya algebras}

Lemma 2.1. a) There exists an Azumaya algebra $\mathcal{A}$ on $\left(\mathbb{A}^{2 n}\right)^{(1)} / \Gamma_{n}$, such that the pull-back of $\mathcal{A}$ to $Y^{(1)}$ is Morita equivalent to $\mathrm{O}$, while its pull-back to $\left(\mathbb{A}^{2 n}\right)^{(1)}$ is $\Gamma_{n}$-equivariantly Morita equivalent to $\mathrm{W}$.

b) The Azumaya algebra $\mathcal{A}$ splits on the formal neighborhood of $L^{(1)} / \Gamma_{n}$.

Proof. (a) is shown in [2, Proposition 6.5] (see also correction below in section 5). We will use another construction of $\mathcal{A}$, appearing in [1] and recalled in the next section 3. (We do not address the question of comparing the two constructions). More precisely, we set $\mathrm{O}=\mathbf{A}_{1,0, . ., 0}$ in the notations of Proposition 3.6 below.

To check (b) we first check that $\left.\mathcal{A}\right|_{L^{(1)} / \Gamma_{n}}$ splits. Since $L^{(1)} / \Gamma_{n}$ is smooth, in view of [19, Corollary IV.2.6] (which says that the Brauer group of a regular scheme injects into the Brauer group of a dense open subscheme) it suffices to check that the restriction of $\mathcal{A}$ to a nonempty open subset in $L^{(1)} / \Gamma_{n}$ splits. Let $L_{0} \subset L$ be the union of free $\Gamma_{n}$ orbits. Then splitting of $\left.\mathcal{A}\right|_{L_{0}^{(1)} / \Gamma_{n}}$ amounts to a $\Gamma_{n}$-equivariant splitting of $\mathrm{W}_{\mathrm{k}}$ on $L_{0}^{(1)}$. This is provided by the module $\mathcal{O}(L)$ equipped with the natural action of $\mathrm{W}_{\mathrm{k}}$, where elements in $L$ act by multiplication by linear functions and elements in a fixed $\Gamma_{n}$-invariant Lagrangian complement ' $L$ act by derivatives. We now check by induction that pull-back of $\mathcal{A}$ to the $n$-th infinitesimal neighborhood of $L^{(1)} / \Gamma_{n}$ splits; moreover, given a choice of a splitting on the $n$-th infinitesimal neighborhood, there exists a compatible splitting on the $(n+1)$ st one, which clearly implies statement (b). The compatible splitting on the $(n+1)$ st neighborhood exists since the obstruction lies in the second cohomology of $\mathcal{O}$ on the affine variety $L^{(1)} / \Gamma_{n}$.

Corollary 2.2. a) The Azumaya algebra $\mathrm{W}_{\mathrm{k}}$ splits $\Gamma_{n}$-equivariantly on $\widehat{U}_{L^{(1)}}^{(1)} ; \widehat{U}_{0}^{(1)}$.

b) The Azumaya algebra $\mathrm{O}$ splits on $\widehat{Y}_{\mathfrak{L}^{(1)}}^{(1)}$.

Now we have the following chain of equivalences of derived categories, cf. [2, Remark 6.8 and the proof of Theorem 6.7]: 


$$
\begin{aligned}
D^{b} \operatorname{Coh}\left(\widehat{Y}_{\mathfrak{L}^{(1)}}^{(1)}\right) \cong D^{b} & \left(\left.\mathrm{O}\right|_{\widehat{Y}_{\mathfrak{L}^{(1)}}^{(1)}}-\operatorname{Mod}\right) \underset{R \Gamma}{\stackrel{\sim}{\longrightarrow}} D^{b}\left(\left.\mathrm{~W}_{\mathrm{k}}^{\Gamma_{n}}\right|_{\widehat{M}_{0}(W, V)_{\mathfrak{L}_{0}^{(1)}}^{(1)}}-\operatorname{Mod}\right) \\
& \cong D^{b}\left(\left.\mathrm{~W}_{\mathrm{k}} \rtimes \mathrm{k}\left[\Gamma_{n}\right]\right|_{\widehat{U}_{L^{(1)}}^{(1)}}-\operatorname{Mod}\right) \cong D^{b} \operatorname{Coh}^{\Gamma_{n}}\left(\widehat{U}_{L^{(1)}}^{(1)}\right)
\end{aligned}
$$

Notice that the first (respectively, the last) equivalence depends on the choice of a splitting bundle whose existence is guaranteed by Corollary 2.2(b) (respectively, a). We denote those splitting bundles by $\mathcal{E}_{\mathcal{L}}, \mathcal{E}_{L}$ respectively.

Proposition 2.3. There exists an equivalence of triangulated categories

$$
\Phi_{\mathbf{k}}: D^{b} \operatorname{Coh}^{\mathbb{G}_{m}^{(1)} \times\left(\mathbb{G}_{m}^{(1)}\right)^{h} \times \Gamma_{n}}\left(U^{(1)}\right) \stackrel{\sim}{\longrightarrow} D^{b} \operatorname{Coh}^{\mathbb{G}_{m}^{(1)} \times\left(\mathbb{G}_{m}^{(1)}\right)^{h}}\left(Y^{(1)}\right),
$$

which is compatible with the composed equivalence in (2.1) for an appropriate choice of the splitting bundles in Corollary 2.2.

Proof. Let $\mathcal{A}$ be as in Lemma 2.1, and let $\mathcal{E}_{\text {res }}$ be a splitting bundle for $\mathrm{O} \otimes_{\mathcal{O}\left(Y^{(1)}\right)} \pi^{*}\left(\mathcal{A}^{o p}\right)$. Then it is shown in [2] that the algebra $\operatorname{End}\left(\mathcal{E}_{r e s}\right)^{o p}$ is Morita equivalent to $\Gamma_{n} \# \mathcal{O}\left(\mathbb{A}^{2 n}\right)$ and the functor $\mathcal{F} \mapsto R \operatorname{Hom}\left(\mathcal{E}_{\text {res }}, \mathcal{F}\right)$ provides an equivalence

$$
D^{b}\left(\operatorname{Coh}\left(Y^{(1)}\right)\right) \stackrel{\sim}{\longrightarrow} D^{b}\left(\operatorname{End}\left(\mathcal{E}_{r e s}\right)^{o p}-\operatorname{Mod}_{f g}\right) \cong \operatorname{Coh}^{\Gamma_{n}}\left(\left(\mathbb{A}^{2 n}\right)^{(1)}\right) .
$$

Here the first equivalence is given by $\mathcal{F} \mapsto R \operatorname{Hom}\left(\mathcal{E}_{r e s}, \mathcal{F}\right)$. To describe the second equivalence we need to fix a $\Gamma_{n}$-equivariant splitting bundle $\mathcal{E}_{\text {orb }}$ for $W_{k}$. Then we get:

$$
\begin{aligned}
& \operatorname{End}\left(\varepsilon_{r e s}\right)=\Gamma(\mathrm{O}) \otimes_{\mathcal{O}\left(\mathbb{A}^{2 n} / \Gamma_{n}\right)^{(1)}} \Gamma(\mathcal{A})=\mathrm{W}^{\Gamma_{n}} \otimes_{\mathcal{O}\left(\mathbb{A}^{2 n} / \Gamma_{n}\right)^{(1)}} \Gamma(\mathcal{A}) \\
& \sim \Gamma_{n} \# \mathrm{~W} \otimes_{\mathcal{O}\left(\mathbb{A}^{2 n} / \Gamma_{n}\right)^{(1)}} \Gamma(\mathcal{A})=\Gamma_{n} \# \operatorname{End}_{\mathcal{O}\left(\mathbb{A}^{2 n}\right)^{(1)}}\left(\varepsilon_{\text {orb }}\right) \sim \Gamma_{n} \# \mathcal{O}\left(\left(\mathbb{A}^{2 n}\right)^{(1)}\right) .
\end{aligned}
$$

Here the first Morita equivalence is given by the bimodule $\mathrm{W} \otimes_{\mathcal{O}\left(\mathbb{A}^{2 n} / \Gamma_{n}\right)^{(1)}}$ $\Gamma(\mathcal{A})$, while the second one comes from the equivalence between the categories of $\Gamma_{n}$-equivariant coherent sheaves on $\left(\mathbb{A}^{2 n}\right)^{(1)}$ and $\Gamma_{n}$-equivariant modules over $\operatorname{End}_{\mathcal{O}\left(\mathbb{A}^{2 n}\right)^{(1)}}\left(\mathcal{E}_{\text {orb }}\right)$ given by $\mathcal{F} \mapsto \mathcal{F} \otimes_{\mathcal{O}} \mathcal{E}_{\text {orb }}$, the latter tensor product being equipped with the diagonal $\Gamma_{n}$-action.

Thus equivalence (2.2) depends on the choice of splitting bundles $\mathcal{E}_{\text {res }}$, $\varepsilon_{\text {orb }}$ in Corollary 2.2, which we assume to be fixed from now on. That choice determines (up to an isomorphism) a choice of splitting bundles $\mathcal{E}_{L}, \mathcal{E}_{\mathcal{L}}$ in the following manner. By Lemma 2.1(b) Azumaya algebra $\mathcal{A}$ splits on the formal neighborhood of $L^{(1)} / \Gamma_{n}$. The splitting bundle on that neighborhood is determined uniquely up to twisting by a line bundle on the formal neighborhood; since every such bundle is easily seen to be trivial, the splitting 
is unique up to an isomorphism. A diagram chase shows that under those choices the equivalences (2.1) and (2.2) are compatible.

We claim that $\varepsilon_{\text {res }}$ carries a canonical $\mathbb{G}_{m}^{(1)} \times\left(\mathbb{G}_{m}^{(1)}\right)^{h}$-equivariant structure. This follows from (the proof of) [2, Proposition 4.3(i)] or Lemma 6.1 below.

The $\mathbb{G}_{m}^{(1)} \times\left(\mathbb{G}_{m}^{(1)}\right)^{h}$-equivariant structure on $\varepsilon_{r e s}$ induces a bigrading on the ring $\operatorname{End}\left(\mathcal{E}_{\text {res }}\right)$, it is easy to see that the category of bigraded modules is canonically equivalent to $\mathrm{Coh}^{\Gamma_{n} \times \mathbb{G}_{m}^{(1)} \times\left(\mathbb{G}_{m}^{(1)}\right)^{h}}\left(\mathbb{A}^{2 n}\right)$.

Thus we get equivalences

$$
\begin{aligned}
D^{b}\left(\operatorname{Coh}^{\mathbb{G}_{m}^{(1)} \times\left(\mathbb{G}_{m}^{(1)}\right)^{h}}\left(Y^{(1)}\right)\right) & \cong D^{b}\left(\operatorname{End}\left(\mathcal{E}_{r e s}\right)^{o p}-\operatorname{Mod}_{\mathrm{fg}}^{\text {bigr }}\right) \\
& \cong D^{b}\left(\operatorname{Coh}^{\Gamma_{n} \times \mathbb{G}_{m}^{(1)} \times\left(\mathbb{G}_{m}^{(1)}\right)^{h}}\left(\mathbb{A}^{2 n}\right)^{(1)}\right),
\end{aligned}
$$

where $\operatorname{Mod}_{\mathrm{fg}}^{\text {bigr }}$ denotes the category of finitely generated bigraded modules.

It is clear from the definition that the constructed equivalence is compatible with (2.2), hence with (2.1).

We will use the same notation $\Phi_{\mathrm{k}}$ for the induced compatible equivalence on categories with less equivariance: $D^{b} \operatorname{Coh}^{\Gamma_{n}}\left(\widehat{U}_{L^{(1)}}^{(1)}\right) \stackrel{\sim}{\longrightarrow} D^{b} \operatorname{Coh}\left(\widehat{Y}_{\mathfrak{L}^{(1)}}^{(1)}\right)$ and $D^{b} \operatorname{Coh}^{\Gamma_{n} \times \mathbb{G}_{m}^{(1)}}\left(\widehat{U}_{L^{(1)}}^{(1)}\right) \stackrel{\sim}{\longrightarrow} D^{b} \operatorname{Coh}^{\mathbb{G}_{m}^{(1)}}\left(\widehat{Y}_{\mathfrak{L}^{(1)}}^{(1)}\right)$.

Remark 2.4. One can construct a $\mathbb{G}_{m}^{(1)} \times\left(\mathbb{G}_{m}^{(1)}\right)^{h}$-equivariant bundle on $Y^{(1)}$ providing an equivalence $\Phi_{\mathrm{k}}$ in a different way which is probably better suited for generalization to other symplectic resolutions of singularities. Instead of Lemma 2.1 one can use Corollary 2.2(b) to get a tilting bundle on the formal neighborhood of $\pi^{-1}(0)$ in $Y^{(1)}$. That bundle can be equipped with a $\mathbb{G}_{m}^{(1)} \times\left(\mathbb{G}_{m}^{(1)}\right)^{h}$-equivariant structure by Proposition 6.3 in the Appendix, which then can be shown to come from a uniquely defined vector bundle on $Y^{(1)}$ (cf. [2, Lemma 2.12]).

Lemma 2.5. a) Assume that the splitting bundles $\mathcal{E}_{\text {res }}, \mathcal{E}_{\mathcal{L}}$ and $\mathcal{E}_{\text {orb }}, \mathcal{E}_{L}$ are chosen compatibly as in the proof of Proposition 2.3.

Then the following diagram is commutative:

$$
\begin{aligned}
& D^{b} \operatorname{Coh}_{\mathfrak{L}^{(1)}}^{\mathbb{G}_{m}^{(1)}}\left(Y^{(1)}\right) \quad \stackrel{\Phi_{k}^{-1}}{\longrightarrow} \quad D^{b} \mathrm{Coh}_{L^{(1)}}^{\mathbb{G}_{m}^{(1)} \times \Gamma_{n}}\left(U^{(1)}\right) \\
& \mathcal{F} \mapsto R \Gamma\left(\mathcal{F} \otimes \mathcal{E}_{\mathcal{L}}\right) \downarrow \quad \mathcal{F} \mapsto \mathcal{F} \otimes \mathcal{E}_{L} \downarrow \\
& D^{b}\left(\mathrm{~W}_{\mathrm{k}}^{\Gamma_{n}}-\operatorname{Mod}_{\mathfrak{L}_{0}^{(1)}}^{\mathbb{G}_{m}^{(1)}}\right) \stackrel{M \mapsto \mathrm{W}_{\mathrm{k}} \otimes_{\mathrm{W}_{\mathrm{k}}^{\Gamma n}} M}{\longrightarrow} D^{b}\left(\mathrm{~W}_{\mathrm{k}} \rtimes \mathrm{k}\left[\Gamma_{n}\right]-\operatorname{Mod}_{L^{(1)}}^{\mathbb{G}_{(1)}^{(1)}}\right),
\end{aligned}
$$

where lower indices denote the set theoretic support condition on the coherent sheaf/module/sheaf of modules. 
b) For any choice of a $\Gamma_{n}$-equivariant splitting $\bar{\varepsilon}_{L}$ for $\mathrm{W}_{\mathrm{k}}$ on $L^{(1)}$ there exists a splitting bundle $\mathcal{E}_{\text {orb }}$ compatible with $\overline{\mathcal{E}}_{L}$.

Proof. a) follows by diagram chase. To check (b) notice that any two $\Gamma_{n^{-}}$ equivariant splitting bundles for $\mathrm{W}_{\mathrm{k}}$ on $L^{(1)}$ differ by a twist by a $\Gamma_{n^{-}}$ equivariant line bundle. It is easy to see that the isomorphism class of such a line bundle is determined by the character of $\Gamma_{n}$ by which it acts on the fiber of the line bundle at zero. Thus we can start with an arbitrary $\mathcal{E}_{\text {orb }}$, after possibly twisting it by a character of $\Gamma_{n}$ we will achieve the desired compatibility.

Notice that $\left.\mathrm{W}\right|_{L^{(1)}}$ has a standard $\Gamma_{n^{-}}$-equivariant splitting provided by the $\mathrm{W}$ module $\mathcal{O}(L)$ where elements in $L \subset U \cong U^{*}$ act by multiplication by linear functions and elements in a fixed $\Gamma_{n}$-invariant complement to $L$ act by derivatives. By Lemma 2.5(b) there exists a splitting bundle $\mathcal{E}_{\text {orb }}$ which is compatible with that equivariant splitting of $\left.\mathrm{W}\right|_{L^{(1)}}$, from now on we assume that the equivalence are defined using a choice of $\varepsilon_{\text {orb }}$ satisfying that compatibility.

Given an irreducible $\mathrm{k}\left[\Gamma_{n}\right]$-module $\rho$, we denote by $\mathcal{F}_{\rho}$ the coherent sheaf $\Phi_{\mathrm{k}}\left(\mathcal{O}_{L^{(1)}} \otimes \rho\right)$.

\subsection{Lagrangian components}

We now fix the Lagrangian linear subspace $L \subset U$ given by the condition of nilpotency of the compositions of $B_{i}$ 's. According to [16, Proposition 7.2.18] or [13, Lemma 5.1], the $\mathbb{G}_{m}^{h}$-fixed points $Y^{\mathbb{G}_{m}^{h}}$ (recall that $Y$ was introduced in Section 2.1) are naturally labeled by $r$-partitions $\boldsymbol{\mu}=\left(\mu^{(1)}, \ldots, \mu^{(r)}\right)$ of total size $|\boldsymbol{\mu}|=\sum_{i}\left|\mu^{(i)}\right|=n$ (recall that $\nu=\nu_{0}+n \delta$ ). We will abuse notation by using the same letter for a multi-partition and for the corresponding fixed point.

Now the Lagrangian subvariety $\mathfrak{L} \subset Y$ is a union of locally closed Lagrangian subvarieties $\mathfrak{L}_{\mu}^{\circ}$ labeled by the set $\mathcal{P}(r, n)$ of $r$-partitions of total size $n$. Each piece $\mathfrak{L}_{\mu}^{\circ}$ is isomorphic to the affine space $\mathbb{A}^{n}$, and is defined as the attracting set of the corresponding $\mathbb{G}_{m}^{h}$-fixed point. We define $\mathfrak{L}_{\boldsymbol{\mu}}$ as the closure of $\mathfrak{L}_{\boldsymbol{\mu}}^{\circ}$. I. Gordon [13, Section 5.4] has defined a partial order on

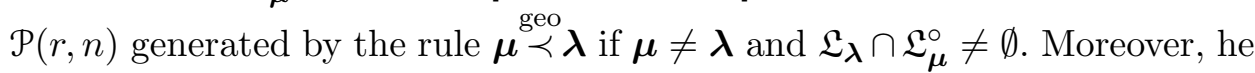
has (at least partly) described this order combinatorially in [13, Sections 6,7]. In order to formulate the description, recall the bijection $\tau_{\mathbf{s}}[13,6.2],[16$, 7.2.17] between the set $\mathcal{P}(r, n)$ of multipartitions, and the set $\mathcal{P}_{\nu_{0}}\left(\left|\nu_{0}\right|+r n\right)$ of usual partitions of size $\left|\nu_{0}\right|+r n$ having $r$-core $\nu_{0}$. Then according to [13, 
Proposition 7.10], we have $\stackrel{\boldsymbol{\mu} \prec \boldsymbol{}}{\text { geo }} \Rightarrow \boldsymbol{\mu} \stackrel{\text { com }}{\prec} \Leftrightarrow{ }^{t} \tau_{\mathbf{s}}\left({ }^{t} \boldsymbol{\mu}\right) \triangleleft{ }^{t} \tau_{\mathbf{s}}\left({ }^{t} \boldsymbol{\lambda}\right)$, that is, one transposed partition dominates the other.

\subsection{Localization for Verma modules for $\Gamma_{n}$-invariants in the Weyl algebra}

According to [2, Theorem 6.3], the functor of global sections $R \Gamma: D^{b}(\mathrm{O}-$ $\bmod ) \rightarrow D^{b}\left(\mathrm{~W}_{\mathrm{k}}^{\Gamma_{n}}-\bmod \right)$ is an equivalence of categories. We denote the quasiinverse equivalence by $M \mapsto{ }^{{ }^{l o c}} M$ (localization).

We define Verma modules for $\mathrm{W}_{\mathrm{k}} \rtimes \mathbb{C}\left[\Gamma_{n}\right]$ by: $\mathrm{V}_{\boldsymbol{\mu}}:=\mathrm{k}[L] \otimes \rho_{\boldsymbol{\mu}}$ where $\boldsymbol{\mu}$ is an $r$-multipartition of $n$, and $\rho_{\boldsymbol{\mu}}$ is the corresponding irreducible $k\left[\Gamma_{n}\right]$ module. Under the Morita equivalence of $\mathrm{W}_{\mathrm{k}} \rtimes k\left[\Gamma_{n}\right]$ and $\mathrm{W}_{k}^{\Gamma_{n}}$, the Verma module $\mathrm{V}_{\boldsymbol{\mu}}$ goes to the module $\mathrm{V}_{\boldsymbol{\mu}}^{\Gamma_{n}}=\mathrm{k}[L]^{\rho_{\mu}}$ where the superscript denotes the $\rho_{\mu}$ isotypic component. The modules $\mathrm{V}_{\boldsymbol{\mu}}^{\Gamma_{n}}$ will be called the spherical Verma modules.

The key step in our argument is provided by the following:

Proposition 2.6. The support $\operatorname{supp}\left({ }^{\operatorname{loc}} \bigvee_{\boldsymbol{\mu}}^{\Gamma_{n}}\right)$ consists of $\mathfrak{L}_{\boldsymbol{\mu}}^{(1)}$ and some $\mathfrak{L}_{\boldsymbol{\lambda}}^{(1)}$ such that $\boldsymbol{\lambda} \stackrel{\operatorname{com}}{\prec} \boldsymbol{\mu}$.

The proof of the Proposition appears at the end of the next section.

\section{Spherical Cherednik algebras and localization in families}

This section is devoted to the proof of Proposition 2.6. In order to carry out the argument we need to include the varieties $Y, \mathbb{A}^{2 n} / \Gamma_{n}$, the $\operatorname{ring} \mathrm{W}_{\mathrm{k}}^{\Gamma_{n}}$ and the sheaf of rings $\mathrm{O}$ in a family, as described in Proposition 3.6.

\subsection{Quiver varieties and their quantization in mixed and positive characteristic}

We start by presenting the usual quiver variety construction in a slightly generalized setting.

We consider an arbitrary quiver $Q$ with the set of vertices $Q_{0}$. We will follow the notations of $[18,4.2]$ for general Nakajima quiver varieties. So $D Q$ is the double quiver, and given $\mathbf{v}, \mathbf{d} \in \mathbb{N}^{Q_{0}}$ we consider a symplectic vector space $R(D Q, \mathbf{v}, \mathbf{d})$ with a symplectic action of $G L(\mathbf{v})$, and its Hamiltonian reduction. More precisely, given a character $\theta: G L(\mathbf{v}) \rightarrow \mathbb{G}_{m}$ (stability), and a central element $\chi \in \mathfrak{z}(\mathfrak{g l}(\mathbf{v}))$ (moment level), we consider the Nakajima quiver variety $\mathfrak{M}_{\chi}^{\theta}(\mathbf{v}, \mathbf{d})$. It is defined in loc. cit. as a complex quasiprojective variety, but its GIT quotient construction works over any commutative ring $\mathrm{R}$ and produces a scheme $\mathfrak{M}_{\chi}^{\theta}(\mathbf{v}, \mathbf{d})_{\mathrm{R}}$. If $\chi$ varies in the family $\mathfrak{z}(\mathfrak{g l}(\mathbf{v}))$, we obtain a family of quiver varieties $\mathfrak{M}_{\mathfrak{z}}^{\theta}(\mathbf{v}, \mathbf{d})_{\mathrm{R}}$ over $\mathfrak{z}(\mathfrak{g l}(\mathbf{v}))_{\mathrm{R}} \simeq \mathbb{A}_{\mathrm{R}}^{Q_{0}}$. 
Furthermore, let $W_{k}^{\hbar}(\mathbf{v}, \mathbf{d})$ denote the asymptotic Weyl algebra of the symplectic vector space $R(D Q, \mathbf{v}, \mathbf{d})$. Thus $\mathrm{W}_{\mathrm{k}}^{\hbar}(\mathbf{v}, \mathbf{d})$ is $\mathrm{k}[\hbar]$-algebra generated by $R(D Q, \mathbf{v}, \mathbf{d})$ subject to the relation $[x, y]=\hbar^{2} \cdot(x, y)$; it can also be described as the Rees algebra of the Weyl algebra $W_{k}(\mathbf{v}, \mathbf{d})$ equipped with Bernstein filtration. The symplectic action of $G L(\mathbf{v})$ on $R(D Q, \mathbf{v}, \mathbf{d})$ gives rise to the map $\tau: \mathfrak{g l}(\mathbf{v}):=\operatorname{Lie} G L(\mathbf{v}) \rightarrow \mathrm{W}_{\mathrm{k}}^{\hbar}(\nu)$. For $\chi$ such that $\chi \cdot \mathbf{v}:=\sum_{i \in Q_{0}} \chi_{i} v_{i}=0$ we set $\tau_{\chi}(\xi):=\tau(\xi)-\hbar^{2} \sum_{i \in Q_{0}} \chi_{i} \operatorname{Tr} \xi_{i}$ where $\xi=\left(\xi_{i}\right)_{i \in Q_{0}} \in \mathfrak{g l}(\mathbf{v})$.

The quantum Hamiltonian reduction is defined as $\mathrm{W}_{\mathrm{k}}^{\hbar}(\mathbf{v}, \mathbf{d}) / /{ }_{\chi} G L(\mathbf{v}):=$ $\left.\left(\mathrm{W}_{\mathrm{k}}^{\hbar}(\mathbf{v}, \mathbf{d})\right) / \mathrm{W}_{\mathrm{k}}^{\hbar}(\mathbf{v}, \mathbf{d}) \cdot \tau_{\chi}(\mathfrak{g l}(\mathbf{v}))\right)^{G L(\mathbf{v})}($ see $[18,4.2])$. Once again, this construction works over any ground ring $\mathrm{R}$ and produces a filtered $\mathrm{R}[\hbar]$-algebra to be denoted $A_{\chi}(\mathbf{v}, \mathbf{d})_{\mathrm{R}}$. This is a specialization of the $\mathrm{R}\left[\mathbb{A}^{Q_{0}}\right]$-algebra $\left.A_{\mathfrak{z}}(\mathbf{v}, \mathbf{d})_{\mathrm{R}}:=\left(\mathrm{W}_{\mathrm{k}}^{\hbar}(\mathbf{v}, \mathbf{d})\right) / \mathrm{W}_{\mathrm{k}}^{\hbar}(\mathbf{v}, \mathbf{d}) \cdot \tau(\mathfrak{s l}(\mathbf{v}))\right)^{G L(\mathbf{v})}$ at the maximal ideal $(\chi)$ in $\mathrm{R}\left[\mathbb{A}^{Q_{0}}\right]$ where $\mathfrak{s l}(\mathbf{v}) \subset \mathfrak{g l}(\mathbf{v})$ is the derived subalgebra.

Finally, recall that one associates with $Q$ a Kac-Moody algebra $\mathfrak{g}_{Q}$, and its weight $\lambda=\sum_{i \in Q_{0}} v_{i} \omega_{i}-\sum_{i \in Q_{0}} d_{i} \alpha_{i}$. The criterion of [5] for the moment map in the Hamiltonian reduction construction of our quiver varieties to be flat takes especially simple form when $\mathfrak{g}_{Q}$ is simple or affine: namely, the flatness follows if $\lambda$ is dominant, see e.g. [3, 2.1.4]. In our application we only need the case of cyclic $\tilde{A}_{r-1}$-quiver $Q$, and $\mathbf{d}=(1,0, \ldots, 0)$. In this case the weight $\lambda$ is dominant iff $\mathbf{v}=n \delta=(n, \ldots, n), n \in \mathbb{N}$.

Lemma 3.1. a) Consider the quiver variety $\mathfrak{M}_{\mathfrak{z}}^{\theta}(\mathbf{v}, \mathbf{d})_{\mathbb{Z}}$ over $\operatorname{Spec} \mathbb{Z}$. There exists a finite localization $\mathrm{R}$ of $\mathbb{Z}$ such that the base change of $\mathfrak{M}_{\mathfrak{z}}^{\theta}(V, W)_{\mathrm{R}}$ to any algebraically closed field $\mathbf{k}$ is the corresponding quiver variety $\mathfrak{M}_{\mathfrak{z}}^{\theta}(\mathbf{v}, \mathbf{d})_{\mathbf{k}}$.

b) Assume that $\mathbf{v}, \mathbf{d}$ are such that the corresponding moment map is flat (e.g. in case $Q$ is Dynkin or extended Dynkin the corresponding weight $\lambda$ is dominant). There exists a finite localization $\mathrm{R}$ of $\mathbb{Z}$, such that the associated graded of the natural filtration on $A_{\mathfrak{z}}(\mathbf{v}, \mathbf{d})_{\mathrm{R}}$ is the ring of functions on the affine quiver variety $\mathfrak{M}_{\mathfrak{z}}^{0}(\mathbf{v}, \mathbf{d})_{\mathrm{R}}$. In other words, the quantum Hamiltonian reduction of the asymptotic Weyl algebra over $\mathrm{R}$ is flat over $\mathrm{R}[\hbar]$.

c) Assume that $\mathbf{v}, \mathbf{d}$ satisfies the assumption of (b) and $R$ satisfies both (a) and (b). Then we have

$$
\begin{aligned}
\operatorname{gr}\left(A_{\mathfrak{z}}(\mathbf{v}, \mathbf{d})_{\mathrm{k}}\right) & =\mathcal{O}\left(\mathfrak{M}_{\mathfrak{z}}^{0}(\mathbf{v}, \mathbf{d})_{\mathrm{k}}\right), \\
\operatorname{gr}\left(A_{\chi}(\mathbf{v}, \mathbf{d})_{\mathrm{k}}\right) & =\mathcal{O}\left(\mathfrak{M}_{\chi}^{0}(\mathbf{v}, \mathbf{d})_{\mathrm{k}}\right),
\end{aligned}
$$

for any field $\mathrm{k}$ with a homomorphism from $R$ and $\chi \in \mathfrak{z}_{\mathrm{k}}^{*}$.

Proof. a) Taking the preimage of $\mathfrak{z}$ under the moment map commutes with any base change. After base change to a finite localization of $\mathbb{Z}$ the categorical 
quotient by the action of $G L(\mathbf{v})$ exists and commutes with any base change by [8, Theorem 33].

b) The quantum Hamiltonian reduction is defined as the module of $G L(\mathbf{v}, \mathrm{R})$ invariants in a quotient of the Weyl algebra by a left ideal. Thus it carries a natural filtration whose associated graded is a submodule in the module of $G L(\mathbf{v}, \mathrm{R})$ invariants of the associated graded of the quotient by the left ideal. As pointed out above, the condition on the weight $\lambda$ implies that the moment map is flat. Flatness of the moment map implies in turn that taking the quotient by the ideal commutes with taking associated graded, thus we see that the associated graded of the filtration on the quantum Hamiltonian reduction is a subring in the classical Hamiltonian reduction. Furthermore, the ambient ring here is finitely generated and the embedding becomes an isomorphism upon base change to $\mathbb{C}$. It follows that the two rings become equal upon base change to a finite localization of $\mathrm{R}$.

c) follows since there is a natural injective map from the left hand side to the right hand side, statements $(a, b)$ together show these maps are surjective.

In the rest of this subsection we work over a fixed field $\mathrm{k}=\overline{\mathrm{k}}$ of characteristic $p>0$.

Recall that for an algebraic variety $X$ over $\mathrm{k}$ we denote by $X^{(1)}$ its Frobenius twist and Fr: $X \rightarrow X^{(1)}$ is the Frobenius morphism.

Lemma 3.2. Assume that $p>\mathbf{v}_{i}$ for all $i$.

a) The ring $A_{\mathfrak{z}}(\mathbf{v}, \mathbf{d})_{\mathrm{k}}$ has a natural structure of a ring over $\mathcal{O}\left(\mathfrak{M}_{\mathfrak{z}}^{0}(\mathbf{v}, \mathbf{d})^{(1)} \times{ }_{\left(\mathbb{A}^{Q_{0}}\right)^{(1)}}\left(\mathbb{A}_{\hbar}^{1} \times \mathbb{A}^{Q_{0}}\right)\right)$, where we used the map $A S_{Q}:\left(\hbar, \chi_{0}\right.$, $\left.\ldots, \chi_{r-1}\right) \mapsto\left(\chi_{0}^{p}-\hbar^{p-1} \chi_{0}, \ldots, \chi_{r-1}^{p}-\hbar^{p-1} \chi_{r-1}\right), \mathbb{A}_{\hbar}^{1} \times \mathbb{A}^{Q_{0}} \rightarrow\left(\mathbb{A}^{Q_{0}}\right)^{(1)}$.

b) Reducing the structure described in (a) at $\hbar=0$ we get a map $\mathcal{O}\left(\mathfrak{M}_{\mathfrak{z}}^{0}(\mathbf{v}, \mathbf{d})^{(1)} \rightarrow \mathcal{O}\left(\mathfrak{M}_{\mathfrak{z}}^{0}(\mathbf{v}, \mathbf{d})\right)\right.$; this map equals pull-back under the Frobenius morphism.

Proof. To check (a) we address following [1, Section 3] the special features of Hamiltonian reduction in positive characteristic.

For a connected smooth linear algebraic group $G$ over k we have an exact sequence of groups $1 \rightarrow G_{1} \rightarrow G \stackrel{\mathrm{Fr}}{\longrightarrow} G^{(1)} \rightarrow 1$ where $G_{1}$ stands for the Frobenius kernel. The Lie algebra $\mathfrak{g}$ of $G$ is equipped with a natural structure of a $p$-Lie algebra, and its universal enveloping algebra $U(\mathfrak{g})$ contains the $p$-center $\mathfrak{Z}(\mathfrak{g})$.

We denote by $\mathbb{X}^{*}(G)$ the lattice of characters $G \rightarrow \mathbb{G}_{m}$ and we set $\mathbb{X}^{*}(\mathfrak{g})=\mathbb{X}^{*}(G) \otimes \mathrm{k}$. We have $\mathbb{X}^{*}(\mathfrak{g}) \subset \mathfrak{g}^{*}$. 
For $\chi \in X^{*}(\mathfrak{g})$ we denote by $I_{\chi}$ the kernel of the corresponding homomorphism $U(\mathfrak{g}) \rightarrow$ k. We set $I_{\chi}^{(1)}:=I_{\chi} \cap \mathfrak{Z}(\mathfrak{g})$, a maximal ideal of $\mathfrak{Z}(\mathfrak{g})$. We let $\mathfrak{u}_{\chi}(\mathfrak{g})$ denote the quotient of $U(\mathfrak{g})$ by the two-sided ideal generated by $I_{\chi}^{(1)}$. The image of $I_{\chi}$ in $\mathfrak{u}_{\chi}(\mathfrak{g})$ is denoted by $\mathfrak{i}_{\chi} \subset \mathfrak{u}(\mathfrak{g})$.

We now take $G=G L(\mathbf{v})$ and we denote its Lie algebra by $\mathfrak{g l}(\mathbf{v})$. Thus $X^{*}(\mathfrak{g})=\mathfrak{z}^{*}$ (where we used the assumption $p>\mathbf{v}_{i}$ ).

The center $\mathfrak{Z}(\mathbf{v}, \mathbf{d})$ of the Weyl algebra $W_{\mathrm{k}}(\mathbf{v}, \mathbf{d})$ equals $\mathrm{k}\left[R(D Q, \mathbf{v}, \mathbf{d})^{(1)}\right]$, while that of $W_{\mathrm{k}}^{\hbar}(\mathbf{v}, \mathbf{d})$ equals $\mathrm{k}\left[\mathbb{A}_{\hbar}^{1} \times R(D Q, \mathbf{v}, \mathbf{d})^{(1)}\right]$.

Thus $W_{\mathrm{k}}^{\hbar}(\mathbf{v}, \mathbf{d})$ can be viewed as a coherent sheaf of algebras over $\mathbb{A}_{\hbar}^{1} \times$ $R(D Q, \mathbf{v}, \mathbf{d})^{(1)}$.

The quantized moment map is the homomorphism $\mathfrak{g l}(\mathbf{v}) \rightarrow W_{\mathrm{k}}^{\hbar}(\mathbf{v}, \mathbf{d})$. Its restriction to $\mathfrak{Z}(\mathfrak{g l}(\mathbf{v}))$ corresponds to the map of spectra $\mu_{\hbar}^{A S}: \mathbb{A}_{\hbar}^{1} \times$ $R(D Q, \mathbf{v}, \mathbf{d})^{(1)} \rightarrow\left(\mathfrak{g l}(\mathbf{v})^{*}\right)^{(1)}$ given by $\mu_{\hbar}^{A S}(\hbar, x)=A S_{Q}(\hbar, \mu(x))$, where $\mu$ : $R(D Q, \mathbf{v}, \mathbf{d}) \rightarrow \mathfrak{g l}(\mathbf{v})^{*}$ is the moment map [24, Proposition 2.1 $1^{\prime}$.

We obtained a map from $\left.\mathcal{O}\left(\mu_{\hbar}^{A S}\right)^{-1}\left(X^{*}(\mathfrak{g})\right) / / G L(\mathbf{v})^{(1)}\right)=$ $\mathcal{O}\left(\mathfrak{M}_{\mathfrak{z}}^{0}(\mathbf{v}, \mathbf{d})^{(1)} \times \mathbb{A}_{\hbar}^{1}\right)$ to the center of the quantum Hamiltonian reduction $\mathfrak{Z}\left(\left(\mathrm{W}_{\mathrm{k}}^{\hbar}(\mathbf{v}, \mathbf{d}) / \operatorname{Im}(\mathfrak{s} l(\mathbf{v}))^{G L(\mathbf{v})}\right)\right.$. We also have a map from $\mathrm{k}[\mathfrak{z}]$ to $\mathfrak{Z}\left(\left(\mathrm{W}_{\mathrm{k}}^{\hbar}(\mathbf{v}, \mathbf{d}) / \operatorname{Im}(\mathfrak{s l}(\mathbf{v}))^{G L(\mathbf{v})}\right)\right.$.

Combining the two maps together we get a map

$$
\mathcal{O}\left(\mathfrak{M}_{\mathfrak{z}}^{0}(\mathbf{v}, \mathbf{d})^{(1)} \times{ }_{\left(\mathbb{A}^{Q_{0}}\right)^{(1)}}\left(\mathbb{A}_{\hbar}^{1} \times \mathbb{A}^{Q_{0}}\right)\right) \rightarrow \mathfrak{Z}\left(\left(\mathrm{W}_{\mathrm{k}}^{\hbar}(\mathbf{v}, \mathbf{d}) / \operatorname{Im}(\mathfrak{s l}(\mathbf{v}))^{G L(\mathbf{v})}\right),\right.
$$

where the fiber product involves the map $\mu_{\hbar}^{A S}$, this follows from the definition of $\mu_{\hbar}^{A S}$. This proves (a).

The specialization of the $p$-center of the Weyl algebra at $\hbar=0$ produces the subring $\mathcal{O}(R(D Q, \mathbf{v}, \mathbf{d}))^{(1)} \stackrel{\mathrm{Fr}^{*}}{\longrightarrow} \mathcal{O}(R(D Q, \mathbf{v}, \mathbf{d}))$. Applying Hamiltonian reduction we get (b).

Remark 3.3. It is not hard to see that at least for large $p$ and $\mathbf{v}, \mathbf{d}$ such that the corresponding moment map is flat, the map from $\mathcal{O}\left(\mathfrak{M}_{\mathfrak{z}}^{0}(\mathbf{v}, \mathbf{d})^{(1)} \times{ }_{\left(\mathbb{A}^{Q_{0}}\right)^{(1)}}\right.$ $\left.\left(\mathbb{A}_{\hbar}^{1} \times \mathbb{A}^{Q_{0}}\right)\right)$ to the center of $A_{\mathfrak{z}}(\mathbf{v}, \mathbf{d})_{\mathbf{k}}$ is an isomorphism.

We now combine Hamiltonian reduction in positive characteristic as recalled in the proof of Lemma 3.2 with the GIT quotient procedure.

More precisely, we consider $\tilde{\mathcal{A}}:=\left(\mathrm{W}_{\mathrm{k}}^{\hbar}(\mathbf{v}, \mathbf{d})^{(1)} / \mathrm{W}_{\hbar}^{\mathrm{k}}(\mathbf{v}, \mathbf{d})^{(1)} \cdot \mathfrak{s l}(\mathbf{v})\right)^{G L(\mathbf{v})_{1}}$. This is a sheaf of associative rings with a $G L(\mathbf{v})^{(1)}$ action. As in the proof of the previous Lemma we have maps from $\mathcal{O}\left(\mu^{-1}\left(X^{*}(\mathfrak{g l}(\mathbf{v}))\right)^{(1)}\right.$ and from $\mathrm{k}[\mathfrak{z} \oplus \mathrm{k} \cdot \hbar]$ to the center $\mathfrak{Z}(\tilde{\mathcal{A}})$; together they yield a $G L(\mathbf{v})^{(1)}$-equivariant map

$$
\mathcal{O}\left(\mu^{-1}\left(X^{*}(\mathfrak{g l}(\mathbf{v}))\right)^{(1)} \times\left(\mathbb{A}^{\left.Q_{0}\right)^{(1)}}\left(\mathbb{A}_{\hbar}^{1} \times \mathbb{A}^{Q_{0}}\right)\right) \rightarrow \mathfrak{Z}(\tilde{\mathcal{A}}) .\right.
$$


Thus we can view $\tilde{\mathcal{A}}$ as a $G L(\mathbf{v})^{(1)}$-equivariant sheaf over $\left.\mu^{-1}\left(X^{*}(\mathfrak{g l}(\mathbf{v}))\right)^{(1)} \times_{\mathbb{A}^{Q_{0}}}\left(\mathbb{A}_{\hbar}^{1} \times \mathbb{A}^{Q_{0}}\right)\right)$.

We now fix a generic (i.e. lying off the walls, see $[23,2.3]$ ) stability parameter $\theta$ and let $\mu^{-1}\left(X^{*}(\mathfrak{g l}(\mathbf{v}))\right)_{\theta} \subset \mu^{-1}\left(X^{*}(\mathfrak{g l}(\mathbf{v}))\right)$ be the subset of $\theta$-stable points. The quotient $\mu^{-1}\left(X^{*}(\mathfrak{g l}(\mathbf{v}))\right)_{\theta} / G L(\mathbf{v})$ is by definition the family of quiver varieties $\mathfrak{M}_{\mathfrak{z}}^{\theta}$. We set $\left(\mathfrak{M}_{\mathfrak{z}}^{\theta}\right)^{A S}=\left(\mu^{-1}\left(X^{*}(\mathfrak{g l}(\mathbf{v}))\right)_{\theta}^{(1)} \times_{\mathbb{A} Q_{0}}\right.$ $\left.\left.\left(\mathbb{A}_{\hbar}^{1} \times \mathbb{A}^{Q_{0}}\right)\right)\right) / G L(\mathbf{v})$, clearly $\left.\left(\mathfrak{M}_{\mathfrak{z}}^{\theta}\right)^{A S}=\mathfrak{M}_{\mathfrak{z}}^{\theta} \times \mathbb{A}^{Q_{0}}\left(\mathbb{A}_{\hbar}^{1} \times \mathbb{A}^{Q_{0}}\right)\right)$.

Lemma 3.4. a) The sheaf $\tilde{\mathcal{A}}$ descends to a sheaf of rings $\mathcal{A}_{\mathfrak{z}}^{\theta}$ over $\left(\mathfrak{M}_{\mathfrak{z}}^{\theta}\right)^{A S}$. The restriction of this sheaf to the open part $\hbar \neq 0$ is an Azumaya algebra, while its restriction to the closed subvariety $\hbar=0$ is isomorphic to $\operatorname{Fr}_{*}(\mathcal{O})$.

b) Assume that $(\mathbf{v}, \mathbf{d})$ satisfy the condition of Lemma 3.1(b) and $p \gg 0$. Then $R \Gamma\left(\mathcal{A}_{\mathfrak{z}}^{\theta}\right)=A_{\mathfrak{z}}(\mathbf{v}, \mathbf{d})$, while for every $\chi$ we have $R \Gamma\left(\mathcal{A}_{\chi}^{\theta}\right)=A_{\chi}(\mathbf{v}, \mathbf{d})$.

Proof. a) The action of $G L(\mathbf{v})$ on $\mu^{-1}\left(X^{*}(\mathfrak{g l}(\mathbf{v}))\right)_{\theta}$ is free. This is well known in characteristic zero (see e.g. [25, Proposition 2.6]), and the case of positive characteristic is checked by a similar argument. ${ }^{2}$

For a free action the factorization is a principal $G$-bundle, so descent theory applies, see [20, Proposition 0.9]. Applying this to $\tilde{\mathcal{A}}$ we get a sheaf of rings on $\left(\mathfrak{M}_{\mathfrak{z}}^{\theta}\right)^{A S}$. The first property is established as in [1] and the second one is clear from the construction.

Under the conditions of (b) the higher direct image of the structure sheaf under the maps $\mathfrak{M}_{\mathfrak{z}}^{\theta} \rightarrow \mathfrak{M}_{\mathfrak{z}}^{0}, \mathfrak{M}_{\chi}^{\theta} \rightarrow \mathfrak{M}_{\chi}^{0}$ vanish: in characteristic zero, this follows from Grauert-Riemmenschneider Theorem, the case of large positive characteristic follows since the support of the higher direct image of $\mathcal{O}$ under the morphism of (the family of) quiver varieties over $\mathbb{Z}$ is a $\mathbb{G}_{m}$-invariant closed subset which does not intersect the fiber over the generic point of $\operatorname{Spec}(\mathbb{Z})$, thus it is contained in the preimage of a finite subject in $\operatorname{Spec}(\mathbb{Z})$.

Hence the higher direct image of $\operatorname{Fr}_{*}\left(\mathcal{O}_{\mathfrak{M}_{z}^{\theta}}\right), \operatorname{Fr}_{*}\left(\mathcal{O}_{\mathfrak{M}_{\chi}^{\theta}}\right)$ also vanish, thus the same is true for $\mathcal{A}$. Thus its global sections is a Cohen-Macaulay $\mathcal{O}_{\mathfrak{M}_{3}^{0-}}$ (resp. $\mathcal{O}_{\mathfrak{M}_{\chi}^{0}}$ ) module. On the open part $U$ where the map $\mathfrak{M}^{\theta} \rightarrow \mathfrak{M}^{0}$ is

${ }^{2}$ In loc. cit. it is checked that the action of $G L(\mathbf{v})$ on $\mu^{-1}\left(X^{*}(\mathfrak{g l}(\mathbf{v}))\right)_{\theta}$ has trivial stabilizers. In general this does not imply that the action is free, see [20, Example 0.4] (recall that an action of $G$ on $X$ is free if the map $\left(a \times p r_{2}\right): G \times X \rightarrow X \times X$ is a closed embedding, where $a$ is the action and $p r_{2}$ is the second projection). However, here we are dealing with an action of $G L(\mathbf{v})=\prod G L\left(\mathbf{v}_{i}\right)$ on a locally closed subvariety in the linear representation $R(D Q, \mathbf{v}, \mathbf{d})$. Given $x, y \in R(D Q, \mathbf{v}, \mathbf{d})$ and $g=\left(g_{i}\right) \in G L(\mathbf{v})$ the equation $g(x)=y$ is linear in the matrix coefficients of $g_{i} \in G L\left(\mathbf{v}_{i}\right)$. By standard linear algebra, locally in $x$ we have an algebraic expression for these matrix coefficients in terms of coordinates of $x, y$ provided that a unique solution exists. This shows that the action is free if the stabilizers are trivial. 
an isomorphism it is isomorphic to the restriction of $A_{\mathfrak{z}}(\mathbf{v}, \mathbf{d})$ (respectively, $A_{\chi}(\mathbf{v}, \mathbf{d})$. This latter algebra is also a Cohen-Macaulay module in view of Lemma 3.1(b). The complement to $U$ has codimension at least two; more precisely, $\mathfrak{M}_{\chi}^{\theta} \rightarrow \mathfrak{M}_{\chi}^{0}$ is semismall, while $\mathfrak{M}_{\mathfrak{z}}^{\theta} \rightarrow \mathfrak{M}_{\mathfrak{z}}^{0}$ is small (this is known in characteristic zero [23], hence in large positive characteristic). Thus we get (b).

\subsection{The family of Cherednik algebras and their localization}

Recall that the algebras $\mathrm{W}_{\mathrm{k}}^{\Gamma_{n}} \subset \mathrm{W}_{\mathrm{k}} \rtimes \mathrm{k}\left[\Gamma_{n}\right]$ fit into the $(r+1)$-parametric family of (spherical) rational Cherednik algebras $e \mathbf{H} e \subset \mathbf{H}$, see e.g. [18, 6.1]. The parameters are denoted by $\hbar, c_{0}, c_{1}, \ldots, c_{r-1}$ (in loc. cit. $c_{0}$ is denoted $k$ ). The case of the Weyl algebra corresponds to $\hbar=1, c_{0}=c_{1}=\ldots=c_{r-1}=0$.

Recall also that a spherical rational Cherednik algebra specialized at $\hbar=$ 0 is commutative, thus we get a family of affine cyclic Calogero-Moser spaces $\mathbf{Z}$, over the space $\mathbb{A}_{c}^{r}$ with coordinates $c_{0}, \ldots, c_{r-1}$, namely $\mathbf{Z}=\operatorname{Spec}(e \mathbf{H} e)$, and the fiber $\mathbf{Z}_{\underline{c}}=\operatorname{Spec}\left(e \mathbf{H}_{(0, \underline{c})} e\right)$.

We now present a description of these algebras as a quantization of an affine quiver variety constructed via quantum Hamiltonian reduction.

In order to be able to apply Lemma 3.1 we use quiver data different from the one in $\S 1.1$. Namely, the smooth quasiprojective Nakajima quiver variety $\mathfrak{M}(V, W)$ is defined as a GIT quotient with respect to the stability vector $\theta=(1, \ldots, 1)$, that is the character of $G_{\nu}=G L(\nu):=\prod_{i \in I} G L\left(V_{i}\right)$ equal to $\theta(\underline{g})=\prod_{i \in I} \operatorname{det}\left(g_{i}\right)^{-1}$. We choose an element $\sigma$ of the $\tilde{A}_{r-1}$ affine Weyl group such that $\sigma\left(\lambda_{0}-n \delta\right)=\lambda_{0}-\nu$, and consider $\theta_{\nu}=\sigma^{-1}(\theta)$. Then the works of Lusztig, Nakajima and Maffei on reflection functors provide an isomorphism $\mathfrak{M}(V, W)=\mathfrak{M}^{\theta}(V, W) \stackrel{\sim}{\longrightarrow} \mathfrak{M}^{\theta_{\nu}}\left(V^{\prime}, W\right)$ where $\underline{\operatorname{dim}} V^{\prime}=n \delta$, and $\operatorname{dim} W=\lambda_{0}=(1,0, \ldots, 0)$, see e.g. [3, $\left.\$ 2.1 .3\right]$ and references therein. These references treat the case of a coefficient field of characteristic zero, but the same argument applies for a field of large positive characteristic.

From now on we will replace the stability condition $\theta$ by $\theta_{\nu}$, and $V \bullet$ by $V_{\bullet}^{\prime}$. Thus we have:

$$
Y=\mathfrak{M}^{\theta_{\nu}}\left(V^{\prime}, W\right)
$$

Lemma 3.5. For an appropriate choice of isomorphism $\mathbb{A}_{c}^{r} \rightarrow \mathbb{A}^{I}, \underline{c} \rightarrow$ $\chi(\underline{c})$, we have $e \mathbf{H}_{(\hbar, \underline{c})} e \cong \mathrm{W}_{\mathrm{k}}^{\hbar}(n \delta) / / / \chi(\underline{c}) G_{n \delta}$.

More precisely, set $\eta=\exp (2 \pi i / r) \in \mathbb{Z}[\sqrt[r]{1}] \subset \mathbb{C}$. Choose a prime ideal $\mathfrak{p} \subset \mathbb{Z}[\sqrt[r]{1}]$ over $p$, and keep the same notation for the reduction of $\eta$ in $\mathbb{Z}[\sqrt[r]{1}] / \mathfrak{p} \subset \overline{\mathbb{F}}_{p}$. 
Then $\chi(\underline{c})_{0}:=c_{0}+r^{-1}\left(1-r-\sum_{m=1}^{r-1} c_{m}\right), \chi(\underline{c})_{l}:=r^{-1}\left(1-\sum_{m=1}^{r-1} \eta^{m l} c_{m}\right)$ for $1 \leq l \leq r-1$.

Proof. A similar statement in characteristic zero is shown in [12, Theorem 3.13]. To deduce the case of positive characteristic we use the following easy general statement. Let $\mathrm{R}$ be a commutative ring finitely generated over $\mathbb{Z}$. Assume that two finitely presented algebras over $\mathrm{R}$ have isomorphic base changes to $\mathbb{C}$; then they become isomorphic after base change to some finitely generated commutative flat R-algebra, in particular their base change to an algebraically closed field of almost any prime characteristic. The two algebras in question are the spherical rational DAHA and the algebra obtained by quantum Hamiltonian reduction from the Weyl algebra over $\mathbb{Z}$.

To make sure they are finitely presented it suffices to check that they (or rather their base change to a finite localization of $\mathrm{R}$ ) admit a filtration with a commutative finitely generated associated graded. For the spherical Cherednik algebra this is clear, as it has a filtration whose associated graded is the ring of $\Gamma_{n}$-invariants in the symmetric algebra. For the quantum Hamiltonian reduction this follows from Lemma 3.1.

We now assume that $\operatorname{char}(\mathbf{k})=p \gg 0$. We let $\mathbf{Z}^{A S}=\mathbf{Z}_{\mathbf{k}}^{(1)} \times_{\left(\mathbb{A}^{r}\right)^{(1)}}\left(\mathbb{A}_{\hbar}^{1} \times\right.$ $\mathbb{A}^{r}$, where the map $A S_{Q}: \mathbb{A}_{\hbar}^{1} \times \mathbb{A}^{r} \rightarrow\left(\mathbb{A}^{r}\right)^{(1)}$ is used.

Proposition 3.6. Assume that char $(\mathrm{k})=p \gg 0$. There exists a smooth family $\mathbf{Y}$ of algebraic varieties over the base $\mathbb{A}^{r+1}=\mathbb{A}_{\hbar}^{1} \times \mathbb{A}_{c}^{r}$ with coordinates $\hbar, c_{0}, c_{1}, \ldots, c_{r-1}$, and a flat sheaf of algebras $\mathbf{A}$ on $\mathbf{Y}^{(1)}$ with the following properties.

(a) The fiber $\mathbf{Y}_{(1,0, \ldots, 0)}$ equals $Y$ (introduced in Section 2.1).

(b) We have a proper map from $\mathbf{Y}$ to $\mathbf{Z}^{A S}$. This map is an isomorphism over an open subset $\left(\mathbf{Z}^{0}\right)^{A S} \subset \mathbf{Z}^{A S}$, which contains the fiber $\mathbf{Z}_{c, \mathbf{k}}$ for a generic $\underline{c}$.

(c) We have $\mathbf{A}_{(0, \underline{c})} \cong \operatorname{Fr}_{*} \mathcal{O}_{\mathbf{Y}_{(0, c)}}$, while the restriction of $\mathbf{A}$ to the open subspace $\hbar \neq 0$ is an Azumaya algebra.

(d) $\Gamma\left(\mathbf{Y}^{(1)}, \mathbf{A}\right)=e \mathbf{H} e$.

(e) The dilation action of $\mathbb{G}_{m}$ on parameters, $t:\left(\hbar, c_{0}, \ldots, c_{r-1}\right) \mapsto$ $\left(t \hbar, t c_{0}, \ldots, t c_{r-1}\right)$ lifts to an action on $\mathbf{Y}$; the sheaf $\mathbf{A}$ admits a natural $\mathbb{G}_{m}$-equivariant structure.

Proof. We let $\mathbf{Y}=\left(\mathfrak{M}_{\mathfrak{z}}^{\theta_{\nu}}\left(n \delta, \lambda_{0}\right)^{A S}\right.$, and we consider the sheaf of algebras $\mathbf{A}=\mathcal{A}_{\mathfrak{z}}^{\theta_{\nu}}$.

Then part (a) follows from (3.3), part (d) from Lemma 3.4(b) and Lemma 3.5, part (c) from Lemma 3.4(a), and (e) is clear from the construction. Part (b) follows from part (d) and Lemma 3.5. 


\subsection{Verma modules}

According to [1, Theorem 7.2.4], the functor of global sections $R \Gamma$ : $D^{b}\left(\mathbf{A}_{\hbar, \chi(\underline{c})}-\bmod \right) \rightarrow D^{b}\left(e \mathbf{H}_{(\hbar, \underline{c})} e-\bmod \right)$ is an equivalence of categories provided that $\mathbf{A}_{\hbar, \underline{c}}$ has finite homological dimension; parameters $(\hbar, \underline{c})$ satisfying this property will be called good.

Good parameters form a dense Zariski open subset in the space of parameters. While it is possible to describe this set explicitly (for large $p$ ) based on results of [6], we will only need the following easily available information: $(\hbar=1, \underline{c}=0)$ is good; for a generic $\underline{c}$ the pair $(\hbar, \underline{c})$ is good for any $\hbar$.

We denote the quasiinverse equivalence by $M \mapsto{ }^{\operatorname{loc}} M$ (localization).

We will apply this notation both for a specific good parameter and for a family of such.

Recall Verma modules for $\mathrm{W}_{\mathrm{k}} \rtimes \mathrm{k}\left[\Gamma_{n}\right]$ and $\mathrm{W}_{k}^{\Gamma_{n}}$ introduced in 2.4. The same construction applied to the Cherednik algebra with variable parameters produces the Verma $\mathbf{H}$-modules $\mathbf{V}_{\boldsymbol{\mu}}$, and the spherical Verma $e \mathbf{H} e$ modules $\mathbf{V}_{\boldsymbol{\mu}}^{\Gamma_{n}}$. The specialization of $\mathbf{V}_{\boldsymbol{\mu}}^{\Gamma_{n}}$ to $(\hbar, \underline{c}) \in \mathbb{A}_{\hbar}^{1} \times \mathbb{A}_{c}^{r}$ will be denoted by $\mathbf{V}_{\boldsymbol{\mu},(\hbar, c)}^{\Gamma_{n}}$ : a spherical Verma module over $e \mathbf{H}_{(\hbar, \underline{c})} e$. So for example $\mathbf{V}_{\boldsymbol{\mu},(1,0, \ldots, 0)}^{\Gamma_{n}}=\mathrm{V}_{\boldsymbol{\mu}}^{\Gamma_{n}}$.

Lemma 3.7. We have ${ }^{\text {loc }} \bigvee_{\boldsymbol{\mu}_{n}}^{\Gamma_{n}} \in \mathbf{A}_{\chi(\underline{0})}-\bmod \subset D^{b}\left(\mathbf{A}_{\chi(\underline{0})}-\bmod \right)$, i.e. ${ }^{{ }^{l o c}} \bigvee_{\mu^{\Gamma_{n}}}$ is an $\mathbf{A}_{\chi(\underline{0})}$-module (as opposed to a complex of modules).

Proof. We choose a complementary $\Gamma_{n}$-invariant Lagrangian subspace ' $L$ : ' $L \oplus L=U$. The composed projection $Y \rightarrow \mathbb{A}^{2 n} / \Gamma_{n} \rightarrow{ }^{\prime} L / \Gamma_{n}=\mathbb{A}^{n} / \Gamma_{n}$ is flat since all the fibers are of the same dimension $n$. Hence a sequence $e_{1}, \ldots, e_{n}$ of generators of $\mathrm{k}\left[\mathbb{A}^{n} / \Gamma_{n}\right]$ is a regular sequence for $\mathcal{O}_{Y}$, hence so is the sequence $e_{1}^{p}, \ldots, e_{n}^{p}$. Thus $e_{1}^{(1)}, \ldots, e_{n}^{(1)}$ is a regular sequence for $\mathrm{Fr}_{*} \mathcal{O}_{Y}$ on $Y^{(1)}$, where $e_{i}^{(1)}$ is the function $e_{i}^{p}$ viewed as a function on $Y^{(1)}$. Using Proposition 3.6(c,e) we see that $e_{1}^{(1)}, \ldots, e_{n}^{(1)}$ is a regular sequence for the Azumaya algebra $\mathrm{O}=\mathbf{A}_{\chi(\underline{0})}$ on $Y^{(1)}$. According to Corollary 2.2, on the formal neighborhood of the central fiber, $\mathbf{A}_{\chi(\underline{0})} \simeq \operatorname{End}(E)$ splits. Since $E$ contains $\mathcal{O}_{Y^{(1)}}$ as a direct summand, $\operatorname{End}(E)$ contains $E$ as a direct summand, hence $e_{1}^{(1)}, \ldots, e_{n}^{(1)}$ forms a regular sequence for $E$ as well.

To finish the proof observe that $\mathrm{V}_{\boldsymbol{\mu}}^{\Gamma_{n}}$ is direct summand in $\mathrm{W}^{\Gamma_{n}} \otimes_{\mathrm{k}}\left[e_{1}, \ldots, e_{n}\right]$ $\mathrm{k}$ : in characteristic zero this is clear since category $\mathcal{O}$ for $c=0$ is semi-simple, while the Morita equivalence $\mathrm{W}^{\Gamma_{n}} \sim \mathrm{W}$ sends $\mathrm{W}^{\Gamma_{n}} \otimes_{\mathrm{k}\left[e_{1}, \ldots, e_{n}\right]} \mathrm{k}$ to $\mathrm{W} \otimes_{\mathrm{k}\left[e_{1}, \ldots, e_{n}\right]}$ $\mathrm{k}=\mathrm{W} \otimes_{\mathrm{k}\left[{ }^{\prime} L\right]}\left(\mathrm{k}\left[{ }^{\prime} L\right] /\left(e_{1}, \ldots e_{n}\right)\right)$ which carries a filtration whose associated graded is a direct sum of Verma modules, each one appearing with a nonzero multiplicity. It follows that $\mathrm{V}_{\boldsymbol{\mu}}^{\Gamma_{n}}$ is direct summand in $\mathrm{W}^{\Gamma_{n}} \otimes_{\mathrm{k}\left[e_{1}, \ldots, e_{n}\right]} \mathrm{k}$ also 
when $\mathrm{k}$ has a large positive characteristic. Now it is clear that $\mathrm{W}^{\Gamma_{n}} \otimes_{\mathbf{k}\left[e_{1}, \ldots, e_{n}\right]}$ $\mathrm{k}=R \Gamma\left(\mathrm{O}_{\otimes^{2}}^{L}\left[e_{1}, \ldots, e_{n}\right] \mathrm{k}\right)$ thus ${ }^{\text {loc }} \mathrm{V}_{\boldsymbol{\mu}}^{\Gamma_{n}}$ is a direct summand in $\mathrm{O}^{L}{ }_{\mathrm{k}\left[e_{1}, \ldots, e_{n}\right]} \mathrm{k}$.

\subsection{Supports of Verma modules}

We need some properties of the bijection between irreducible $\Gamma_{n}$ modules and $\left(\mathbb{G}_{m}\right)^{2}$-fixed points, which will be presently deduced from the results of [11]. In fact, loc. cit. establishes similar results for some spherical Cherednik algebras over a field of zero characteristic. Passage between the present setting and that of loc. cit. is done in several steps, which schematically can be depicted as follows:

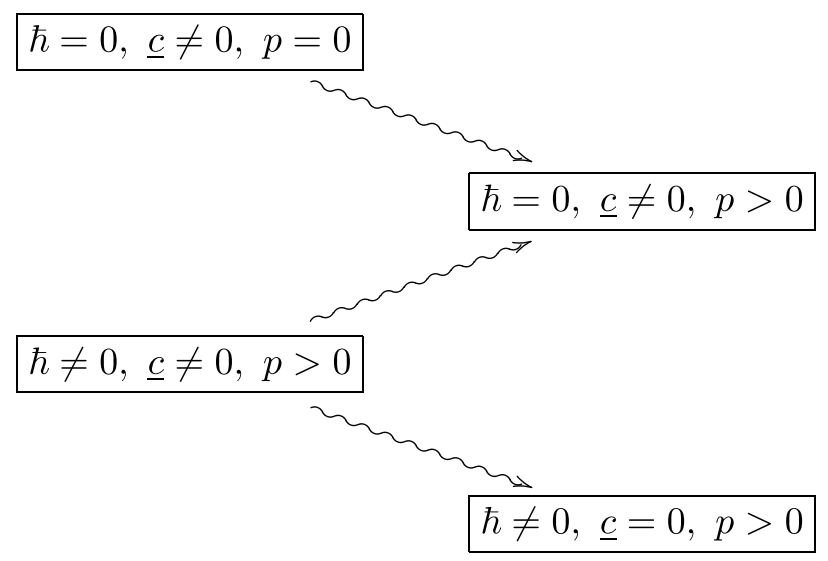

Here the first two degenerations are analyzed in Lemma 3.9, while the last one will be treated using Lemmas 3.7, 3.8.

We start with a geometric Lemma on the behavior of our Lagrangian subvarieties under the degeneration $\underline{c} \rightarrow 0$; it will be applied for varieties over a field of characteristic $p \gg 0$.

The hyperbolic $\mathbb{G}_{m}^{h}$ of Section 2.3 acts on $\mathbf{Y}$, and the $\mathbb{G}_{m}^{h}$-fixed points in a general fiber are uniformly numbered by the set of $r$-partitions of $n$, according to [13, 3.8-3.10]. As in Section 2.3, we define $\mathfrak{L}_{\boldsymbol{\lambda}, \underline{\underline{c}}}^{\circ} \subset \mathbf{Z}_{\underline{c}}$ as the attracting set of the corresponding $\mathbb{G}_{m}^{h}$-fixed point. Contrary to the situation of Section 2.3, for a general ( $\underline{c}$ ) the Lagrangian subvariety $\mathfrak{L}_{\lambda, \underline{c}}^{\circ} \subset \mathbf{Z}_{\underline{c}}$ is closed, i.e. equals its closure $\mathfrak{L}_{\lambda, \underline{\underline{c}}}$.

As $\underline{c}$ goes to $\underline{0}$, the Lagrangian subvariety $\mathfrak{L}_{\mu, \underline{c}}$ degenerates into the (closed) Lagrangian subvariety contained in $\mathfrak{L}_{\boldsymbol{\mu}} \cup \bigcup_{\boldsymbol{\lambda}}^{{ }^{\text {com }} \boldsymbol{\mu}} \mathfrak{L}_{\boldsymbol{\lambda}}$. In effect, let 
$\mathbf{L}_{\boldsymbol{\mu}} \subset \mathbf{Y}$ be the closed irreducible subvariety of $\mathbf{Y}$ whose fiber over general

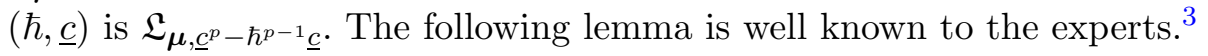

Lemma 3.8. The intersection $\mathbf{L}_{\boldsymbol{\mu}} \cap Y$ contains $\mathfrak{L}_{\boldsymbol{\mu}} \cup \bigcup_{\boldsymbol{\lambda}}{ }_{\text {geo }} \mathfrak{L}_{\boldsymbol{\lambda}}$ and is contained in $\mathfrak{L}_{\boldsymbol{\mu}} \cup \bigcup_{\boldsymbol{\lambda} \sim \boldsymbol{\mu}}^{\operatorname{com}} \mathfrak{L}_{\lambda}$.

Proof. The intersection $\mathbf{L}_{\boldsymbol{\mu}} \cap Y$ is Lagrangian in $Y$ since it is contained in $\pi^{-1} \mathfrak{L}_{0}=\mathfrak{L}$. Since $\mathbf{L}_{\mu} \cap Y$ is closed and contains $\mathfrak{L}_{\mu}$, it must contain $\mathfrak{L}_{\boldsymbol{\mu}} \cup \bigcup_{\boldsymbol{\lambda}}^{\text {geo }} \mathfrak{L}_{\boldsymbol{\mu}}$. Conversely, if $\mathfrak{L}_{\boldsymbol{\lambda}} \subset \mathbf{L}_{\boldsymbol{\mu}} \cap Y$, then $\boldsymbol{\lambda} \stackrel{\text { com }}{\prec \boldsymbol{\mu}}$. To see this let us consider first the case $r=1$ (no cyclic group). Then both the geometric and combinatorial orders are nothing but the dominance order on partitions. The parameter $\underline{c}$ is just one number $c$, and $\mathbf{Y}$ is just the family degenerating the Calogero-Moser space into the Hilbert scheme. The equivariant Borel-Moore étale homology groups $H_{B M}^{\mathbb{G}_{m}^{h}}\left(\mathbf{Y}_{(\hbar, c)}\right)$ are canonically identified for all the fibers of $\mathbf{Y}$. The fundamental classes of $\mathfrak{L}_{\mu, c^{p}-\hbar^{p-1} c}$ form a basis in $H_{B M}^{\mathbb{G}_{m}^{h}}\left(\mathbf{Y}_{(\hbar, c)}\right)$. For $c^{p}-\hbar^{p-1} c \neq 0$, when $\mathfrak{L}_{\mu, c^{p}-\hbar^{p-1} c}$ is closed, its fundamental class in the localized equivariant Borel-Moore homology is proportional to the class of the fixed point $\mu$. On the other hand, under the above identification, this class equals a class in $H_{B M}^{\mathbb{G}_{m}^{h}}(Y)$ supported in $\mathbf{L}_{\mu} \cap Y$ (and proportional to the fixed point class). Now under the well known identification of $H_{B M}^{\mathbb{G}_{m}^{h}}(Y)$ with the degree $n$ part of the Fock space of symmetric functions, the fixed point classes correspond to the Schur functions, while the fundamental classes of $\mathfrak{L}_{\lambda}$ correspond to the monomial functions (see e.g. [22]). The transition matrix between these two bases is upper triangular in the dominance order, hence we are done.

For general $r$, recall that $Y=Y_{\Gamma, \nu}$ is a connected component of the fixed point set $\left(\operatorname{Hilb}^{m}\left(\mathbb{A}^{2}\right)\right)^{\Gamma}$ where $m=v_{0}+\ldots+v_{r-1}$. A fixed point $\boldsymbol{\lambda} \in Y$ goes to the fixed point ${ }^{t} \tau_{\mathbf{s}} \mathbf{( \lambda )}$ (a partition of $m$; notations of 2.3) of $\operatorname{Hilb}^{m}\left(\mathbb{A}^{2}\right)$. If $\boldsymbol{\lambda}$ lies in $\mathbf{L}_{\boldsymbol{\mu}} \cap Y$, then ${ }^{t} \tau_{\mathbf{s}}(\boldsymbol{\lambda})$ lies in $\mathbf{L}_{\tau_{\mathbf{s}}(\boldsymbol{\mu})} \cap \operatorname{Hilb}^{m}\left(\mathbb{A}^{2}\right)$, hence ${ }^{t} \tau_{\mathbf{s}}(\boldsymbol{\lambda}) \triangleleft{ }^{t} \tau_{\mathbf{s}}(\boldsymbol{\mu})$, that is $\boldsymbol{\lambda} \stackrel{\text { com }}{\preceq}$.

Lemma 3.9. For a general $(\underline{c})$, the support $\operatorname{supp}\left({ }^{\operatorname{loc}} \mathbf{V}_{\boldsymbol{\mu},(\hbar, \underline{c})}^{\Gamma_{n}}\right)$ equals $\mathfrak{L}_{\boldsymbol{\mu},\left(\underline{c}^{p}-\hbar^{p-1} \underline{c}\right)} \cdot$

Proof. As the Verma module $\mathbf{V}_{\boldsymbol{\mu},(\hbar, \underline{c})}$ as well as its spherical counterpart $\mathbf{V}_{\boldsymbol{\mu},(\hbar, \underline{c})}^{\Gamma_{n}}$ is indecomposable, the support $\operatorname{supp}\left({ }^{{ }^{l o c}} \mathbf{V}_{\boldsymbol{\mu},(\hbar, \underline{c})}^{\Gamma_{n}}\right)$ must be contained in exactly one component $\mathfrak{L}_{\boldsymbol{\lambda},\left(\underline{c}^{p}-\hbar^{p-1} \underline{c}\right)}^{(1)}$. It is easy to see that dimension of

\footnotetext{
${ }^{3}$ We thank Ben Webster who explained it to us.
} 
the support equals $n$ which is also the dimension of $\mathfrak{L}_{\boldsymbol{\lambda},\left(\underline{c}^{p}-\hbar^{p-1} \underline{c}\right)}^{(1)}$, thus

$$
\operatorname{supp}\left({ }^{\operatorname{loc}} \mathbf{V}_{\boldsymbol{\mu},(\hbar, \underline{c})}^{\Gamma_{n}}\right)=\mathfrak{L}_{\boldsymbol{\lambda},\left(\underline{c}^{p}-\hbar^{p-1} \underline{c}\right)}^{(1)} .
$$

It remains to prove that $\boldsymbol{\lambda}=\boldsymbol{\mu}$. It follows from the last displayed equality that any finite dimensional $\mathbb{G}_{m}^{h}$-equivariant quotient of $\mathbf{V}_{\boldsymbol{\mu},(\hbar, \underline{c})}$ is supported at the point $\boldsymbol{\lambda}$ of the spectrum of the $p$-center of the Cherednik algebra $\mathbf{H}_{(\hbar, \underline{c})}$. On the other hand, $\mathbf{V}_{\boldsymbol{\mu},(0, \underline{c})}$ is the fiber over a prime over $p$ in Spec $\mathbb{Z}[\sqrt[r]{1}]$ of the family of Verma modules over the family of Cherednik algebras. Recall the family $\mathbf{M}(\boldsymbol{\mu})$ of baby Verma modules over the family of Cherednik algebras over Spec $\mathbb{C} \rightarrow \operatorname{Spec} \mathbb{Z}[\sqrt[r]{1}]$ introduced in $[11,4.1]$. This family of Cherednik algebras comes from one over $\operatorname{Spec} \mathbb{Z}[\sqrt[r]{1}]$, and its fiber over a prime over $p$ coincides with the family $\mathbf{H}_{(0, \underline{c}), \mathrm{k}}$ of Section 3.2. The corresponding family of spherical Verma (resp. baby Verma) modules is denoted $\mathbf{V}_{\boldsymbol{\mu},(0, \underline{c}), \mathrm{k}}^{\Gamma_{n}}\left(\operatorname{resp} . \mathbf{M}(\boldsymbol{\mu})_{\underline{c}, \mathrm{k}}^{\Gamma_{n}}\right)$. Now $\mathbf{V}_{\boldsymbol{\mu},(0, \underline{c}), \mathrm{k}}^{\Gamma_{n}}$ surjects onto the spherical baby Verma module $\mathbf{M}(\boldsymbol{\mu})_{c, \mathrm{k}}^{\Gamma_{n}}$. The support of $\mathbf{M}(\boldsymbol{\mu})_{c, \mathrm{k}}^{\Gamma_{n}}$ as a module over the $\hbar=0$ spherical algebra $e \overline{\mathbf{H}}_{(0, \underline{c})} e=\mathrm{k}\left[\mathbf{Z}_{\underline{c}}\right]$ is the fixed point $\boldsymbol{\mu}$. Recall that the $p$-center of the spherical algebra $e \mathbf{H}_{(\hbar, \underline{c}), \mathrm{k}} e$ equals $\mathrm{k}\left[\mathbf{Z}_{\left.\underline{c}^{p}-\hbar^{p-1} \underline{c}\right)}^{(1)}\right]$, and the map from the spectrum of the $p$-center to the spectrum of the $\hbar=0$ spherical algebra is the Frobenius map (Lemma 3.5(c)), hence the $p$-support of $\mathbf{M}(\boldsymbol{\mu})_{\underline{c}, \mathrm{k}}^{\Gamma_{n}}$ is also the fixed point $\boldsymbol{\mu}$. It follows that $\boldsymbol{\lambda}=\boldsymbol{\mu}$. This completes the proof of the lemma along with the proof of the proposition.

Proof. of Proposition 2.6. Recall the family of quiver varieties $\varrho: \mathbf{Y} \rightarrow$ $\mathbb{A}_{\hbar}^{1} \times \mathbb{A}_{c}^{r}$ introduced in Proposition 3.6. We need to show that

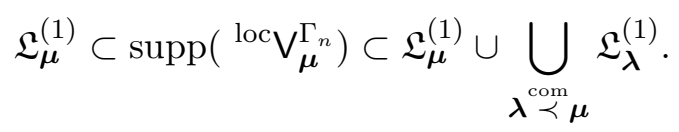

In view of Lemmas 3.8 and 3.9 this would follow once we check that

i) the support of $\operatorname{supp}\left({ }^{\operatorname{loc}} \mathbf{V}_{\boldsymbol{\mu}^{n}}^{\Gamma_{n}}\right)$ is irreducible and

ii) $\operatorname{supp}\left(\operatorname{loc}^{\Gamma_{\boldsymbol{\mu}}}\right)=\operatorname{supp}\left({ }^{\operatorname{loc}} \mathbf{V}_{\boldsymbol{\mu}}^{\Gamma_{n}}\right) \cap \varrho^{-1}(1,0, \ldots, 0)$.

Now, ${ }^{\text {loc }} \mathbf{V}_{\boldsymbol{\mu}}^{\Gamma_{n}}$ is an object in the derived category of sheaves of modules over the Azumaya algebra A concentrated in non-positive homological degrees. Clearly, ${ }^{l o c} \mathbf{V}_{\boldsymbol{\mu}}^{\Gamma_{n}}$ is obtained from ${ }^{{ }^{\circ}} \mathbf{V}_{\boldsymbol{\mu}}^{\Gamma_{n}}$ by derived tensor product with skyscraper at $\hbar=1, \underline{c}=0$ over the ring of functions $\mathrm{k}\left[\mathbb{A}_{\hbar}^{1} \times \mathbb{A}_{c}^{r}\right]$. Lemma 3.7 shows that the latter tensor product is concentrated in homological degree zero. It follows that in some Zariski neighborhood of the spe- 
cial fiber $\varrho^{-1}(1,0, \ldots, 0)$ the complex ${ }^{{ }^{l o c}} \mathbf{V}_{\boldsymbol{\mu}}^{\Gamma_{n}}$ is concentrated in homological degree zero and is torsion free as a module over the ring of functions $\mathrm{k}\left[\mathbb{A}_{\hbar}^{1} \times \mathbb{A}_{c}^{r}\right]$. Thus every component of $\operatorname{supp}\left({ }^{{ }^{\circ o c}} \mathbf{V}_{\mu^{n}}^{\Gamma_{n}}\right)$ whose closure intersects $\varrho^{-1}(1,0, \ldots, 0)$ maps dominantly to $\mathbb{A}_{\hbar}^{1} \times \mathbb{A}_{c}^{r}$. Since the support is $\mathbb{G}_{m}$-invariant under the $\mathbb{G}_{m}$-action of Proposition 3.6(e), property (i) is established. Since ${ }^{\text {loc }} \boldsymbol{V}_{\boldsymbol{\mu}}^{\Gamma_{n}}={ }^{\text {loc }} \mathbf{V}_{\boldsymbol{\mu}}^{\Gamma_{n}} \otimes_{\mathrm{k}\left[\mathbb{A}_{\hbar}^{1} \times \mathbb{A}_{c}^{r}\right]} \mathrm{k}\left[\varrho^{-1}(1,0, \ldots, 0)\right]$, property (ii) is also clear.

\section{The proof of the main Theorem}

\subsection{Procesi bundle}

Under the equivalence $\Phi_{\mathrm{k}}$ of $(2.1)$ the structure sheaf $\mathcal{O}_{U^{(1)}} \rtimes \mathrm{k}\left[\Gamma_{n}\right] \in$ $D^{b} \operatorname{Coh}^{\mathbb{G}_{m} \times \Gamma_{n}}\left(U^{(1)}\right)$ goes to $\mathcal{E} \in D^{b} \operatorname{Coh}_{m}^{\mathbb{G}}\left(Y^{(1)}\right)$, to be called the Procesi vector bundle. It splits into the direct $\operatorname{sum} \mathcal{E}=\bigoplus \mathcal{E}_{\boldsymbol{\lambda}} \otimes \rho_{\boldsymbol{\lambda}}^{*}$ over the set of $r$-partitions of $n$ numbering the irreducible $\mathrm{k}\left[\Gamma_{n}\right]$-representations; under the above equivalence $\mathcal{E}_{\boldsymbol{\lambda}}$ corresponds to $\mathcal{O}_{U^{(1)}} \otimes \rho_{\boldsymbol{\lambda}} \in D^{b} \mathrm{Coh}^{\mathbb{G}_{m} \times \Gamma_{n}}\left(U^{(1)}\right)$.

Recall that for an irreducible $\mathrm{k}\left[\Gamma_{n}\right]$-module $\rho_{\boldsymbol{\lambda}}$ we denote by $\mathcal{F}_{\boldsymbol{\lambda}}$ the coherent sheaf $\Phi_{\mathrm{k}}\left(\mathcal{O}_{L^{(1)}} \otimes \rho_{\boldsymbol{\lambda}}\right)$.

We denote by ${ }^{\prime} L \subset U$ the $\Gamma_{n}$-invariant Lagrangian complement to $L$ in $U$. Repeating the definitions of Section 2.3 with $L$ replaced by ${ }^{\prime} L$ we obtain the Lagrangian components ${ }^{\prime} \mathfrak{L}_{\mu} \subset Y$ numbered by the $r$-partitions of $n$. Note that the adjacency order on these components is the inverse of the order in loc. cit.: ' $\mathfrak{L}_{\boldsymbol{\lambda}} \cap{ }^{\prime} \mathfrak{L}_{\boldsymbol{\mu}}^{\circ} \neq \emptyset$ iff $\boldsymbol{\lambda}_{\mathfrak{g}}^{\text {geo }} \boldsymbol{\mu}$.

We define the opposite Verma modules over $\mathrm{W}_{\mathrm{k}} \rtimes \mathrm{k}\left[\Gamma_{n}\right]$ as $\mathcal{V}_{\mu}:=$ $\mathrm{k}\left[{ }^{\prime} L\right] \otimes \rho^{*} \boldsymbol{\mu}$. Under the Morita equivalence they correspond to the $\mathrm{W}_{\mathrm{k}}^{\Gamma_{n}}$ modules $\bigvee^{\Gamma_{n}}:=\mathrm{k}\left[{ }^{\prime} L\right]^{\rho t_{\mu}}$. We have $\operatorname{Ext}\left(\vee_{\boldsymbol{\lambda}}, \mathcal{V}_{\boldsymbol{\mu}}\right)=0$ iff $\boldsymbol{\lambda} \neq \boldsymbol{\mu}$, and $\operatorname{Ext}^{n}\left(\mathrm{~V}_{\boldsymbol{\lambda}}, \mathrm{V}_{\boldsymbol{\lambda}}\right) \simeq \mathrm{k}$, since $\operatorname{Ext}_{k[U]}^{\bullet}\left(\mathrm{k}[L], \mathrm{k}\left[{ }^{\prime} L\right]\right)$ is a one-dimensional $\mathrm{k}$-vector space in degree $n$, and $\Gamma_{n}$ acts on it via the sign representation $\operatorname{det} L$, and $\rho_{\boldsymbol{\mu}} \otimes \operatorname{det} L \simeq \rho^{t} \boldsymbol{\mu}$.

Also, we define the sheaf ' $\mathcal{F}_{\boldsymbol{\mu}}:=\Phi_{\mathrm{k}}\left(\mathcal{O}_{{ }^{\prime} L^{(1)}} \otimes \rho^{t} \boldsymbol{\mu}\right)$.

Proposition 4.1. We have $\operatorname{supp} \mathcal{F}_{\boldsymbol{\lambda}}=\operatorname{supp}\left({ }^{{ }^{\circ o c}} \mathrm{~V}_{\boldsymbol{\lambda}}\right)$.

Proof. Recall that the $\Gamma_{n}$ equivariant splitting bundle $\mathcal{E}_{\text {orb }}$ used in the definition of the equivalence $\Phi_{\mathrm{k}}$ is assumed to satisfy a compatibility stated after the proof of Lemma 2.5 (end of section 2.2). It is clear that a Verma module $\bigvee_{\boldsymbol{\lambda}}$ is isomorphic to $\mathcal{O}(L) \otimes_{\mathcal{O}_{L^{(1)}}}\left(\mathcal{O}_{L^{(1)}} \otimes \rho_{\boldsymbol{\lambda}}\right)$. Thus the Proposition follows from the commutative diagram in Lemma 2.5(a). 
Corollary 4.2. If the Lagrangian component $\mathfrak{L}_{\boldsymbol{\mu}}^{(1)}$ lies in the support of $\mathcal{F}_{\boldsymbol{\lambda}}$, then $\boldsymbol{\mu} \preceq \boldsymbol{c o m}$. If the Lagrangian component ' $\mathfrak{L}_{\boldsymbol{\mu}}^{(1)}$ lies in the support of ' $\mathcal{F}_{\boldsymbol{\lambda}}$, then $\boldsymbol{\mu} \succeq^{\mathrm{com}} \boldsymbol{\lambda}$.

Proof. We argued in the proof of Proposition 2.6 that the support of the localized Verma module ${ }^{\text {loc}} \mathrm{V}_{\boldsymbol{\lambda}}$ consists of $\mathfrak{L}_{\boldsymbol{\lambda}}^{(1)}$ and some smaller Lagrangian components $\mathfrak{L}_{\boldsymbol{\mu}}^{(1)}, \boldsymbol{\mu} \stackrel{\text { com }}{\prec}$. Thus Corollary follows from Proposition 4.1.

The second part is proved similarly.

\subsection{Proof of Theorem 1.1}

Note that the composed projection $Y \rightarrow \mathbb{A}^{2 n} / \Gamma_{n} \rightarrow \mathbb{A}^{n} / \Gamma_{n}$ is flat since all the fibers are of the same dimension $n$. It follows that $\bar{\varepsilon}_{\rho_{\mu}} \cong \mathcal{F}_{\boldsymbol{\mu}}$ and $\overline{\mathcal{E}}_{\rho_{\boldsymbol{\mu}}}^{\prime} \cong{ }^{\prime} \mathcal{F}_{\boldsymbol{\mu}}$; the required properties of $\mathcal{F}_{\boldsymbol{\mu}},{ }^{\prime} \mathcal{F}_{\boldsymbol{\mu}}$ have been established in Corollary 4.2.

\subsection{Wreath Macdonald polynomials}

The equivalence $\Phi_{\mathrm{k}}$ extends to the same named equivalence $D^{b} \operatorname{Coh}^{T}\left(U^{(1)}\right) \stackrel{\sim}{\longrightarrow} D^{b} \operatorname{Coh}^{T}\left(Y^{(1)}\right)$ where $T$ is the 2-dimensional torus with coordinates $(q, t)$ acting on $\mathbb{A}^{2}$ such that $\mathbb{G}_{m}$ is the diagonal subtorus $\mathbb{G}_{m}=\{(q, q)\}$, while $\mathbb{G}_{m}^{h}$ is the antidiagonal subtorus $\left\{\left(q, q^{-1}\right)\right\}$. We keep the same name for the induced isomorphism of the $K$-groups of the categories in question. We identify the equivariant $K$-group $K^{T}(p t)$ with $\mathbb{Z}\left[q^{ \pm 1}, t^{ \pm 1}\right]$. We denote by $\iota_{\lambda}^{*}: K^{T}\left(Y^{(1)}\right) \rightarrow K^{T}(p t)$ the fiber at the torus fixed point $\boldsymbol{\lambda} \in Y^{(1)}$.

Note that $\iota_{\boldsymbol{\mu}}^{*} \mathcal{E}$ carries an action of $\Gamma_{n}$ since $\mathcal{E}$ carries a fiberwise action of $\Gamma_{n}$ by construction. Thus $\left[\iota_{\mu}^{*} \mathcal{E}\right] \in \mathbb{Z}\left[q^{ \pm 1}, t^{ \pm 1}\right]\left[K\left(\Gamma_{n}\right)\right]$.

Corollary 4.3. $\iota^{*}\left[\mathcal{\mu}[\mathcal{E}] \otimes \sum_{i}(-q)^{i}\left[\Lambda^{i}\left({ }^{\prime} L^{(1)}\right)\right] \in \mathbb{Z}\left[q^{ \pm 1}, t^{ \pm 1}\right]\left[\rho_{\boldsymbol{\lambda}},{ }^{t} \boldsymbol{\lambda} \succeq \boldsymbol{\mu}\right] ;\right.$ also, $\iota_{\boldsymbol{\mu}}^{*}[\mathcal{E}] \otimes \sum_{i}(-t)^{i}\left[\Lambda^{i}\left(L^{(1)}\right)\right] \in \mathbb{Z}\left[q^{ \pm 1}, t^{ \pm 1}\right]\left[\rho_{\boldsymbol{\lambda}},{ }^{t} \boldsymbol{\lambda} \succeq{ }^{\mathrm{com}} \boldsymbol{\mu}\right]$.

Proof. (cf. [16, Proof of Proposition 5.4.2]). The Koszul resolution shows that $\left[\mathcal{O}^{\prime} L^{(1)}\right]=\left[\mathcal{O}_{U^{(1)}}\right] \otimes \sum_{i}(-t)^{i}\left[\Lambda^{i}\left(L^{(1)}\right)\right]$. We have $\operatorname{Tor}^{\mathcal{O}_{L}(1)}\left(\iota_{\boldsymbol{\mu}}^{*}(\mathcal{E}), \mathrm{k}\right)=$ $\operatorname{Tor}^{\mathcal{O}_{U^{(1)}}}\left(\iota_{\boldsymbol{\mu}}^{*}\left(\mathcal{E} \otimes \mathcal{O}^{\prime} L^{(1)}\right), \mathrm{k}\right)$. By the local duality, the second statement of the corollary is equivalent to $\operatorname{Ext}_{\mathcal{O}_{U^{(1)}} \rtimes \mathrm{k}\left[\Gamma_{n}\right]}\left(\mathcal{O}^{\prime} L^{(1)} \otimes \rho_{\boldsymbol{\lambda}}, \iota_{\boldsymbol{\mu}}^{*}(\mathcal{E})\right)=0$ unless $\boldsymbol{\lambda}{ }^{\mathrm{com}}{ }^{t} \boldsymbol{\mu} \Leftrightarrow{ }^{t} \boldsymbol{\lambda} \preceq \boldsymbol{\mu}$. Since $\Phi_{\mathrm{k}}\left(\iota_{\boldsymbol{\mu}}^{*}(\mathcal{E})\right)$ is the skyscraper sheaf at the point $\boldsymbol{\mu} \in$ $Y$, the latter Ext-vanishing follows from supp $\Phi_{\mathrm{k}}\left(\mathcal{O}^{\prime} L^{(1)} \otimes \rho_{\boldsymbol{\lambda}}\right) \subset \bigcup_{\boldsymbol{\mu}}{ }^{\text {com }}{ }^{t}{ }_{\boldsymbol{\lambda}}{ }^{\prime} \mathfrak{L}_{\boldsymbol{\mu}}$.

The first statement of the corollary is proved similarly. 


\section{Errata to $[2]$}

We use this opportunity to correct some mistakes in [2].

Proof of Lemma 2.12 on p. 8: the action of $\mathbb{G}_{m}$ on $X_{R}$ induces an action of the formal completion of $\mathbb{G}_{m}$ on the formal completion $X$, cf. Appendix (section 6). So references to $\mathbb{G}_{m}$-equivariant structures on the bundle on $X$ in the proof should be replaced by ones to equivariance with respect to the formal completion of $\mathbb{G}_{m}$.

Proposition 3.8 on p. 12: "restriction to the generic fiber of the formal scheme $\hat{X}$ " should be removed, the statement of the Proposition should read as follows: "The localization $\mathcal{O}_{\hbar} \otimes_{\mathrm{k}[[\hbar]]} \mathrm{k}((\hbar))$ is a sheaf of Azumaya algebras over the sheaf of commutative rings $\mathcal{O}_{\hat{X}} \otimes_{\mathrm{k}[[\hbar]]} \mathrm{k}((\hbar))$."

The most essential correction ${ }^{4}$ is for section 5.1 .3 on p. 20 which provides the central step in the proof of Proposition 5.3. It is claimed there that the quantization of Kleinian singularity whose existence is claimed in Proposition 5.3 is obtained from the Weyl algebra of the vector space $M$ by Hamiltonian reduction, using the quantum moment map described in Example 5.7. This is false; e.g. in the example $\Gamma=\{ \pm 1\}$ acting on the two dimensional symplectic vector space, (the fiber at $\hbar=1$ of) the quantization arising from Example 5.7 is identified with the enveloping algebra $U(\mathfrak{s l}(2, \mathrm{k}))$ reduced at the singular Harish-Chandra central character $-\rho=-1$, while $\Gamma$ invariants in the Weyl algebra are isomorphic to the reduction of $U(\mathfrak{s l}(2, \mathbf{k}))$ by the regular central character $-\frac{1}{2}$. The argument breaks down as the displayed isomorphism $\mathcal{R}_{\hbar} \cong \mathcal{O}_{\hbar} \otimes \mathcal{O}_{X_{0}}^{p} \otimes \mathcal{O}_{V_{0}}^{p}$ does not hold, because, contrary to the mistaken statement in [2], the commutative ring $\mathcal{O}\left(V^{(1)}\right)[[\hbar]]$ does not act on $\mathcal{R}_{\hbar}$ in a natural way.

In order to get the quantization claimed in Proposition 5.3 one has to use a different quantum moment map, which differs from the one in [2, Example 5.7] by adding the character described in the next paragraph, this fact goes back to $[17]^{5}$ in the case of characteristic zero, the case of large positive characteristic follows by the argument in the proof of Lemma 3.5.

Consider an extended Dynkin quiver with the set of vertices $I$ and the extending vertex $i_{0} \in I$. We set $\delta_{i}, i \in I$, to be the $i$-th coordinate of the corresponding minimal imaginary root vector (e.g. $\left.\delta_{i_{0}}=1\right)$. We choose an

\footnotetext{
${ }^{4}$ We thank Ivan Losev for pointing this out.

${ }^{5}$ Also the following special case of this fact coincides with the special case $n=1$ of Lemma 3.5: we consider the quiver of type $\widehat{A_{r-1}}$, so $I=\mathbb{Z} / r \mathbb{Z}, i_{0}=0$, the orientation is chosen so that $i$ is connected to $i+1$ by an oriented edge for $i \in I$; $\delta_{i}=1 \forall i \in I$, thus $\partial_{i}=0 \forall i \in I$.
} 
orientation of the extended Dynkin quiver. We define the defect vector $\partial$ with coordinates $\partial_{i}$ as follows: $\partial_{i}:=-\delta_{i}+\sum \delta_{j}$ where the sum is over the set of vertices $j$ such that there is an arrow $i \rightarrow j$. Finally, $\Gamma \subset S L(2)$ is the finite subgroup corresponding to our quiver. Then the character producing the Hamiltonian reduction equal to the $\Gamma$-invariants in the first Weyl algebra has the following components: then $\chi_{i_{0}}=\delta_{i_{0}} /|\Gamma|-\partial_{0}-1, \chi_{i}=\delta_{i} /|\Gamma|-\partial_{i}$ $\left(i \neq i_{0}\right)$.

\section{Appendix: Equivariant structure on rigid vector bundles ${ }^{6}$}

Let $\mathbb{T}=\left(\mathbb{G}_{m}\right)^{d}$ be a $d$-dimensional torus. We begin with the following result.

Lemma 6.1. Let $X$ be a proper (not necessarily smooth) scheme over an algebraically closed field $\mathrm{k}, E$ a vector bundle over $X$ such that $\operatorname{Ext}^{1}(E ; E)=$ 0 . Assume that $X$ is endowed with a $\mathbb{T}$-action $m: \mathbb{T} \times X \rightarrow X$. Then the action of $\mathbb{T}$ lifts to $E$, i.e. $E$ has a $\mathbb{T}$-equivariant structure.

Proof. First, we claim that $E$ is $\mathbb{T}$-invariant, that is $m^{*} E \simeq p_{2}^{*} E$ locally with respect to $\mathbb{T}$.

Indeed, since $\operatorname{Ext}^{1}(E ; E)=0$, the claim is true if we replace $\mathbb{T}$ by its formal completion at 1 . Now, let us consider the vector bundle $F=$ $\operatorname{Hom}\left(m^{*} E, p_{2}^{*} E\right)$. Since $X$ is proper, $p_{1 *} F$ is a coherent sheaf on $\mathbb{T}$. Theorem on Formal Functions implies that the formal completion of $p_{1 *} F$ at 1 is a vector bundle. Therefore $p_{1 *} F$ is a vector bundle over some open neighborhood $U$ of 1 in $\mathbb{T}$. Further, shrinking $U$, we may assume that there exists a section $s$ of $p_{1 *} F$ over $U$ which is equal to $\operatorname{Id} \in\left(p_{1 *} F\right)_{1}$ at the point $1 \in \mathbb{T}$. It implies that $m^{*} E \simeq p_{2}^{*} E$ over some open neighborhood of 1 . Since the subgroup of $\mathbb{T}$ generated by an open neighborhood of 1 coincides with $\mathbb{T}$, the claim follows.

We conclude that there exists an extension of affine algebraic groups

$$
1 \rightarrow \operatorname{Aut}(E) \rightarrow G \rightarrow \mathbb{T} \rightarrow 1
$$

such that the action of $G$ on $X$ (through $\mathbb{T}$ ) lifts to $E$.

Note that the group $\operatorname{Aut}(E)$ is smooth and connected (since it is an open subscheme of the affine space $\operatorname{End}(E))$. Hence the lemma follows from the next one.

Lemma 6.2. For a smooth connected group $H$, any extension

$$
1 \rightarrow H \rightarrow G \stackrel{h}{\rightarrow} \mathbb{T} \rightarrow 1
$$

\footnotetext{
${ }^{6}$ By Vadim Vologodsky.
} 
splits in the weak sense, i.e. there exists a homomorphism $i: \mathbb{T} \rightarrow G$ such that $h \circ i=\mathrm{Id}$.

Proof. The result is well known. We include the argument for the readers' convenience.

Any affine algebraic group is a semidirect product of a reductive group and a unipotent one. Therefore it is enough to prove the lemma in the following two cases.

1. $H$ is a unipotent group. In this case the lemma follows from the structural result cited above: if $G=U \times \mathbb{T}$ is the semidirect factorization, the morphism $h$ factors through the second factor and, since the kernel of $h$ is connected, there exists a splitting.

2. $H$ is a reductive group. In this case, $\operatorname{rk}(G)=\operatorname{rk}(H)+\operatorname{dim}(\mathbb{T})$. Therefore, the intersection of a maximal torus $T \subset G$ with $H$ is a toral subgroup of $H$ of dimension $\operatorname{rk}(H)$. Hence its connected component is a Cartan subgroup of $H$; the intersection $T \cap H$ is contained in the centralizer of that Cartan subgroup, hence it coincides with the Car$\tan$ subgroup. Thus, the lemma reduces to the obvious case when $G$ is a torus.

Let $x \rightarrow y$ be a morphism of schemes of finite type over an algebraically closed field. Let $y_{l}$ (resp. $\hat{y}$ ) be the $l$-th infinitesimal neighborhood (resp. the formal neighborhood) of a closed point $y \in \mathcal{y}$, and let $x_{y_{l}}$ (resp. $x_{\hat{y}}$ ) be the preimage of $y_{l}$ (resp. $\hat{y}$ ) in $X$. Assume that $X_{y_{l}},(l=1,2, \cdots)$, are endowed with compatible $\mathbb{T}$-actions $m_{l}: \mathbb{T} \times X_{y_{l}} \rightarrow X_{y_{l}}{ }^{7}$. We will need a version of the notion of an equivariant bundle for formal neighborhoods. By a $\mathbb{T}$ equivariant structure on a vector bundle $E$ over $X_{\hat{y}}$ we mean a compatible system of $\mathbb{T}$-equivariant structures on $E_{l}:=E_{\mid x_{y_{l}}}$. Equivalently, this is an action of $\hat{\mathbb{T}}$ on $E$ lifting the $\hat{\mathbb{T}}$-action on $X_{\hat{y}}$ such that for each $l$ the $\hat{\mathbb{T}}$-action on $E_{l}$ can be extended to an action of $\mathbb{T}$.

Proposition 6.3. Assume that $x \rightarrow y$ is proper and that $\operatorname{Ext}^{1}(E, E)=0$. Then $E$ admits a $\mathbb{T}$-equivariant structure.

Proof. Consider the projective system of algebraic groups $H_{l}=\operatorname{Aut}\left(E_{l}\right)$. Since $\operatorname{End}_{\mathrm{k}}\left(E_{l}\right)$ is a finite-dimensional k-vector space, the projective system above satisfies the Mittag-Leffler condition, i.e., for any integer $l$ there exists an integer $N \geq l$ such that

$$
\operatorname{Im}\left(H_{k} \rightarrow H_{l}\right)=\operatorname{Im}\left(H_{N} \rightarrow H_{l}\right), \quad \forall k>N .
$$

${ }^{7}$ In particular, $X_{\hat{y}}$ is equipped with an action of the formal completion $\hat{\mathbb{T}}$ of $\mathbb{T}$. 
Similarly, using that $\operatorname{Ext}^{1}(E, E)=0$ and that $\operatorname{Ext}^{1}\left(E_{l}, E_{l}\right)$ is finite-dimensional, we conclude that

$$
\operatorname{Im}\left(\operatorname{Ext}^{1}\left(E_{N}, E_{N}\right) \rightarrow \operatorname{Ext}^{1}\left(E_{l}, E_{l}\right)\right)=0,
$$

for $N$ large enough.

Using the above assertions and arguing as in the proof of Lemma 6.1, we conclude that, for every $l$, the bundle $E_{l}$ admits a $\mathbb{T}$-equivariant structure.

Next, consider a projective system of extensions

$$
1 \rightarrow H_{l} \rightarrow G_{l} \rightarrow \mathbb{T} \rightarrow 1,
$$

where, for a scheme $S, G_{l}(S)$ is the group of pairs $(t: S \rightarrow \mathbb{T}, \theta)$, where $\theta$ is an isomorphism between $\left(m_{l} \circ(t \times I d)\right)^{*} E_{l}$ and the pullback of $E_{l}$ on $S \times X_{y_{l}}$ with respect to the second projection. The Mittag-Leffler property of $H_{l}$ together with Lemma 6.2 ensures the existence of a weak splitting $\mathbb{T} \rightarrow \underset{\longleftarrow}{\longleftarrow} G_{l}$ which defines a $\mathbb{T}$-equivariant structure on $E$.

\section{References}

[1] R. Bezrukavnikov, M. Finkelberg, V. Ginzburg, Cherednik algebras and Hilbert schemes in characteristic p, Representation Theory 10 (2006), 245-298.

[2] R. Bezrukavnikov, D. Kaledin, McKay equivalence for symplectic resolutions of quotient singularities, Proc. Steklov Inst. Math. 246, no. 3 (2004), 13-33.

[3] R. Bezrukavnikov, I. Losev, Etingof conjecture for quantized quiver varieties, arXiv:1309.1716.

[4] R. Bezrukavnikov, S. Riche, Affine braid group actions on derived categories of Springer resolutions, Annales Scientifiques de l'Ecole Normale Supérieure 45 (2012), 535-599.

[5] W. Crawley-Boevey, Geometry of the moment map for representations of quivers, Comp. Math. 126 (2001), 257-293.

[6] C. Dunkl, S. Griffeth, Generalized Jack polynomials and the representation theory of rational Cherednik algebras, Selecta Math. (N.S.) 16 (2010), 791-818.

[7] P. Etingof, W. L. Gan, V. Ginzburg, A. Oblomkov, Harish-Chandra homomorphisms and symplectic reflection algebras for wreath-products, Publ. Math. Inst. Hautes Études Sci. 105 (2007), 91-155. 
[8] V. Franjou, W. van der Kallen, Power reductivity over an arbitrary base, Doc. Math. Extra volume: Suslin (2010), 171-195.

[9] W. L. Gan, V. Ginzburg, Almost-commuting variety, D-modules, and Cherednik algebras, with an appendix by Ginzburg. Int. Math. Res. Pap. IMRP 2006, 26439, 1-54.

[10] V. Ginzburg, Isospectral commuting variety and the Harish-Chandra D-module, Duke Math. J. 161 (2012), no 11, 2023-2111.

[11] I. Gordon, Baby Verma modules for rational Cherednik algebras, Bull. London Math. Soc. 35 (2003), 321-336.

[12] I. Gordon, A remark on rational Cherednik algebras and differential operators on the cyclic quiver, Glasg. Math. J. 48 (2006), 145-160.

[13] I. Gordon, Quiver varieties, category $\mathcal{O}$ for rational Cherednik algebras, and Hecke algebras, Int. Math. Res. Pap. IMRP 2008, no. 3, Art. ID rpn006, 69 pp.

[14] I. Gordon, Macdonald positivity via the Harish-Chandra D-module, Inv. Math. 187 (2012) no. 3, 637-643.

[15] M. Haiman, Hilbert schemes, polygraphs and the Macdonald positivity conjecture, J. Amer. Math. Soc. 14 (2001), no. 4, 941-1006.

[16] M. Haiman, Combinatorics, symmetric functions, and Hilbert schemes, in: Current developments in mathematics, 2002, Int. Press, Somerville, MA (2003), 39-111.

[17] M. Holland, Quantization of the Marsden-Weinstein reduction for extended Dynkin quivers, Ann. Sci. de l'É.N.S. $4^{e}$ série 32 (1999), no. 6, 813-834.

[18] I. Losev, Isomorphisms of quantizations via quantization of resolutions, Advances in Mathematics 231 (2012), 1216-1270.

[19] J.-S. Milne, Étale cohomology, Princeton Math. Series 33, Princeton U. Press (1980).

[20] D. Mumford, J. Fogarty, F. Kirwan, Geometric invariant theory. Third edition, Ergebnisse der Mathematik und ihrer Grenzgebiete, 34. Springer-Verlag, Berlin (1994), xiv+292pp.

[21] H. Nakajima, Lectures on Hilbert schemes of points on surfaces, University Lecture Series 18, AMS, Providence, RI (1999).

[22] H. Nakajima, Jack polynomials and Hilbert schemes of points on surfaces, arXiv:alg-geom/9610021. 
[23] H. Nakajima, Quiver varieties and branching, SIGMA 5 (2009), 003, 37 pages.

[24] R. Travkin, Quantum geometric Langlands correspondence in positive characteristic: the $G L_{N}$ case, arXiv/1110.5707.

[25] D. Yamakawa, Geometry of multiplicative preprojective algebra, Int. Math. Res. Pap. IMRP 2008, Art. ID rpn008, 77pp.

Roman BezRukaVnikov

Department of Mathematics

Massachusetts Institute of Technology

CAmbridge MA 02139

USA

National Research University Higher School of Economics INTERNATIONAL LABORATORY OF REPRESENTATION THEORY AND Mathematical Physics

20 MYASNITSKAYA ST.

Moscow 101000

RUSSIA

E-mail address: bezrukav@math.mit.edu

Michael FinKelberG

National Research University Higher School of Economics Department of Mathematics, AND IITP

20 MYASNITSKAYA ST.

Moscow 101000

Russia

E-mail address: fnklberg@gmail.com

VADIM VOLOGODSKY

DePARTMENT OF MATHEMATics

OREGON UNIVERSITY

EUGENE OR 97403

USA

E-mail address: vvologod@uoregon.edu

Received December 18, 2013 